\title{
DECOMPOSITION OF SYMMETRIC POWERS OF IRREDUCIBLE REPRESENTATIONS OF SEMISIMPLE LIE ALGEBRAS AND THE BRION POLYTOPE
}

\author{
A. V. SMIRNOV
}

\begin{abstract}
To any closed irreducible $G$-invariant cone in the space $V$ of a finitedimensional representation of a semisimple Lie group there corresponds a convex polytope called the Brion polytope. This is closely connected with the action of the group $G$ on the algebra of functions on the cone, and also with the moment map. In this paper we give a description of Brion polytopes for the spaces $V$ themselves and for their nullcones.
\end{abstract}

\section{Contents}

1. Introduction

2. The moment map

3. The local structure theorem and a description of $\operatorname{Bri}(V)$ for the adjoint representation

4. Description of $\operatorname{Bri}(V)$ for most irreducible representations

5. Inner bounds and a description of the inner boundary of $\operatorname{Bri}(\mathcal{N}(V))$ for representations with highest weight belonging to the root lattice

6. Description of $\operatorname{Bri}(\mathcal{N}(V))$

\section{INTRODUCTION}

Let $G$ be a simply connected semisimple complex Lie group, and let $G: V$ be a finitedimensional irreducible complex algebraic representation of $G$. Let $X \subset V$ be an affine $G$-invariant cone-manifold. Denote by $\mathbb{C}[X]_{n}$ the space of algebraic functions of degree $n$ on $X$, and by $\mathbb{C}[X]$ the space of all algebraic functions on $X$. We have $S^{n} V=\mathbb{C}\left[V^{*}\right]_{n}$, where $V^{*}$ is the dual of $V$. Taking the direct sum of these equations over $n$, we write $S \cdot(V)=\mathbb{C}\left[V^{*}\right]$.

Let $G: \mathfrak{g}$ be the adjoint representation. We then have the identity

$$
\mathbb{C}[\mathfrak{g}]=\mathbb{C}[\mathfrak{g}]^{G} \otimes \mathbb{C}[\mathcal{N}]
$$

of $G$-modules, where $\mathbb{C}[\mathfrak{g}]^{G}$ is the algebra of invariants, and $\mathcal{N}=\mathcal{N}(\mathfrak{g}) \subset \mathfrak{g}$ is the nullcone (the manifold of nilpotent elements of $\mathfrak{g}$ ); see [14].

Furthermore, in the case of the adjoint representation the algebra $\mathbb{C}[\mathcal{N}]$ is known to split into irreducible $G$-modules (apart from the grading). Each representation appears in this decomposition with multiplicity equal to the dimension of the space of zero weight in it (see [14]). If we take the grading into account, the problem rapidly becomes more complicated. Some information can be found in the paper of Kostant [14], but in order to use this we have to know the multiplicities of the eigenvalues of a certain element of a torus, which itself is a nontrivial problem. Moreover, there exists the formula of Hesselink

2000 Mathematics Subject Classification. Primary 20G05, 22E46, 53D20. 
(see [13]) for the multiplicity $m_{\lambda, n}$ of the representation $V_{(\lambda)}$ with highest weight $\lambda$ in $\mathbb{C}[\mathcal{N}]_{n}$, but to use it is unrealistic in practice since it requires the repeated application of Kostant's formula (see for example [12]).

The problem of splitting a representation of $G$ on the algebra $\mathbb{C}[\mathcal{N}]$ into irreducibles can be formulated not only in the case of the adjoint representation. However, when $V \neq \mathfrak{g}$ the situation becomes even more complicated. The best result in this area is in my opinion the paper of Brion [7]. Let the cone $X$ be irreducible, and let $m_{\lambda, n}(X)$ be the multiplicity of $V_{(\lambda)}$ in $\mathbb{C}[X]_{n}$. Let

$$
\operatorname{Bri}(X)=\left\{x \in X(T) \otimes_{\mathbb{Z}} \mathbb{Q} \mid \exists n \in \mathbb{Z}_{+}, \lambda \in X(T): x=\lambda / n, m_{\lambda^{*}, n} \neq 0\right\},
$$

where $T$ is a maximal torus of $G$, with $X(T)$ the set of its weights, and $\lambda^{*}$ the weight dual to $\lambda$.

We notice immediately that all the highest weights of the representation $G: V$ lie in this set. In the paper cited above it is shown that $\operatorname{Bri}(X)$ is a convex polytope that we shall call the Brion polytope. If the assumption on irreducibility of the cone $X$ is relaxed, then this coincides with the union of the Brion polytopes of the irreducible components, so that it need not be convex. The description of the Brion polytope for the nullcone is a good approximation to a description of the representation of $G$ on the graded algebra of functions on $V$. It is precisely this description that is our problem.

The problem of describing the Brion polytopes of the closures of all nilpotent orbits in the adjoint representation (and thus also of the representation itself) was posed by Popov in [16. In the paper of Broer 9] there can be found a description of the Brion polytopes for all nilpotent $G$-invariant closed cones in the adjoint representation of the group $G_{2}$.

We introduce some terminology and notation that we shall need in order to formulate the results.

Let $\mathfrak{t}$ be a fixed Cartan subalgebra of $\mathfrak{g}$, let $\Delta$ be the root system of the pair $(\mathfrak{g}, \mathfrak{t})$, let $\Delta^{+} \subset \Delta$ be the set of positive roots with respect to a fixed ordering on $\Delta$, and let $\Pi$ be the system of simple roots. It is well known that an irreducible representation of $G$ is uniquely determined by its highest weight, and this in turn can be expressed as a linear combination of the fundamental weights $\pi_{i}$ with nonnegative integer coefficients. These coefficients are called the indices of the highest weight.

Now let $P(V)$ be the projectivization of $V$, and $P(X) \subset P(V)$ the projectivization of the cone $X$.

We choose a compact real form $K$ of the group $G$ such that $T \cap K$ is a maximal torus in $K$. Let $\mathfrak{k}$ be the Lie algebra of the group $K$, and let $(\cdot, \cdot)$ be a $K$-invariant Hermitian scalar product on $V$. Consider the moment map $\mu: P(X) \rightarrow \mathfrak{k}^{*}$. This is well known to be given by the formula

$$
\mu(\langle x\rangle)(a)=\frac{(x, a x)}{2 \pi i(x, x)} .
$$

The dominant Weyl chamber $\mathcal{C}$ is contained in the space $\mathbb{E}:=\mathfrak{t}(\mathbb{R})^{*}=i \operatorname{Lie}(T \cap K)^{*}$, and the set $i \mu(P(X))$ in the space $i \mathfrak{k}^{*}$. Consider the intersection $i \mu(P(X)) \cap \mathbb{E}$, and also $i \mu(P(X)) \cap \mathcal{C}$. In the above-mentioned work of Brion it is proved that

$$
i \mu(P(X)) \cap \mathcal{C}=\operatorname{Bri}(X) .
$$

This gives an alternative approach to describing the Brion polytope. In particular, it is an immediate consequence of this equality that $\operatorname{Bri}(X) \subset \mathcal{C} \cap M(V)$, where $M(V):=$ $\operatorname{conv} \Phi(V)$, and $\Phi(V)$ is the set of weights of the representation $G: V$. Such an approach was used by Sjamaar [17], who described the polytopes $\operatorname{Bri}(V)$ for certain spaces $V$ (we shall discuss this in more detail below). Moreover, the use of moment maps reduces the study of the Brion polytope for semisimple groups to that for simple groups. 
We now turn to stating the results of the present paper.

Let $G: \mathfrak{g}$ be the adjoint representation. It has nontrivial invariants, and hence according to (1.1) we have $0 \in \operatorname{Bri}(\mathfrak{g})$. Moreover, this formula has the stronger consequence:

$$
\operatorname{Bri}(\mathfrak{g})=\operatorname{conv}(\operatorname{Bri}(\mathcal{N}), 0) \text {. }
$$

Next we introduce the notions of inner and outer boundaries of a polytope $P$ contained in an arbitrary vector space:

$$
\begin{aligned}
& P_{\text {int }}=\{x \in P \mid r x \notin P \forall r \subset \mathbb{R}, 0<r<1\} \quad \text { if } 0 \notin P, \quad \text { and } \quad P_{\text {int }}=\{0\} \text { otherwise; } \\
& P_{\text {ext }}=\{x \in P \mid r x \notin P \forall r \subset \mathbb{R}, r>1\} .
\end{aligned}
$$

The faces of the polytope that lie in the inner and outer boundaries are also called inner and outer, respectively. It follows from the formula given above that

$$
\operatorname{Bri}(\mathfrak{g})_{\text {int }}=\{0\} \quad \text { and } \operatorname{Bri}(\mathfrak{g})_{\text {ext }}=\operatorname{Bri}(\mathcal{N})_{\text {ext }} .
$$

It is well known that the nullcone of the adjoint representation is irreducible, and therefore its Brion polytope is convex. The convexity implies that knowledge of its inner and outer boundaries determines it completely. Thus the problem splits into two independent ones: describe the outer boundary of $\operatorname{Bri}(\mathfrak{g})$ and the inner boundary of $\operatorname{Bri}(\mathcal{N})$.

Using the method of moment maps, we can construct some of the points of the Brion polytope. If we could obtain bounds on the polytope coinciding with the convex hull of these points, then we would have a precise description. All the bounds that we shall obtain can be divided into two types. In both cases there will be a certain set $R \subset \mathbb{E}$ that intersects every ray from the origin lying in the Weyl chamber exactly once. Bounds of the form $\operatorname{Bri}(X) \subset \mathbb{R}_{\geq 1} R$ will be called inner bounds, and those of the form $\operatorname{Bri}(X) \subset \mathbb{R}_{\leq 1} R$ outer bounds, where $\mathbb{R}_{\geq 1} R\left(\mathbb{R}_{\leq 1} R\right)$ is the set obtained by multiplying the points of $R$ by the numbers greater (less) than or equal to unity.

In order to obtain outer bounds we use the construction of Grosshans [11] and a Kostant cascade (this construction will be elaborated in Section 3). The Grosshans construction was independently devised by Brion, Luna and Vust (local structure theorem: see [8]) and used by Brion in his paper [7] in order to describe the structure of the Brion polytope in the neighborhood of a point. This description was in turn used by Sjamaar [17] to obtain a certain bound on $\operatorname{Bri}(V)$. In fact the application of the local description is an unnecessary step, and in our view a more direct route is the immediate application of the Grosshans construction which can be carried out repeatedly, thereby constructing a Kostant cascade. This allows us to describe the outer boundary of the Brion polytope for the adjoint representations of the algebras $A_{1}, A_{2}, A_{3}, B_{n}, C_{n}, D_{n}, G_{2}, F_{4}, E_{7}$ and $E_{8}$. Unfortunately, for the cases $A_{\geq 4}$ and $E_{6}$ these bounds are insufficient, and another method is needed.

In addition, we have obtained the following result: the inner boundary of the Brion polytope for the nullcone of the adjoint representation is the intersection of the dominant Weyl chamber and the affine subspace spanned by the simple roots. The vertices of this polytope are the points $c_{i} \pi_{i}$, where $c_{i}$ is the inverse of the sum of the elements of the $i$ th column of the inverse of the Cartan matrix. The description of the vertices of the outer boundary of the Brion polytope for the nullcone of the adjoint representation is given in Table 1 for the algebras indicated above.

In the case $V \neq \mathfrak{g}$ the problem of describing the Brion polytope for the nullcone becomes rapidly more complicated. To begin with, the decomposition $\mathbb{C}[V]=\mathbb{C}[V]^{G} \otimes$ $\mathbb{C}[\mathcal{N}(V)]$, where $\mathcal{N}(V) \subset V$ is the nullcone (the manifold of nilpotent elements in $V)$, is no longer valid. However, if the action has nontrivial invariants, the equality $\operatorname{Bri}(V)=\operatorname{conv}(\operatorname{Bri}(\mathcal{N}(V)), 0)$ does remain valid. The biggest problem, though, is related to the fact that the nullcone may be reducible and accordingly the Brion polytope 
TABLE 1.

\begin{tabular}{|l|l|}
\hline $\mathbb{C} G$ & Vertices of the outer boundary of Bri $(\mathfrak{g})$ \\
\hline$A_{1}$ & $2 \pi_{1}$ \\
\hline$A_{2}$ & $\pi_{1}, \pi_{2}, \pi_{1}+\pi_{2}$ \\
\hline$A_{3}$ & $\pi_{1}+\pi_{3}, 2 \pi_{1} / 3,2 \pi_{3} / 3,\left(2 \pi_{1}+\pi_{2}\right) / 3,\left(2 \pi_{3}+\pi_{2}\right) / 3, \pi_{2}$ \\
\hline$B_{2}$ & $\pi_{1}, 2 \pi_{2}$ \\
\hline$B_{n}$ for $n>2$ & $\pi_{1}, \pi_{2}, \pi_{3} / 2, \pi_{4} / 2, \ldots, \pi_{n-1} /[n / 2], \pi_{n} /[(n+1) / 2]$ \\
\hline$C_{n}$ for $n>2$ & $2 \pi_{1}, 2 \pi_{2} / 2,2 \pi_{3} / 3, \ldots, 2 \pi_{n-1} /(n-1), 2 \pi_{n} / n$ \\
\hline$D_{n}$ for $n / 2 \in \mathbb{Z}$ & $\pi_{1}, \pi_{2}, \pi_{3} / 2, \pi_{4} / 2, \ldots, \pi_{n-3} /((n-2) / 2), \pi_{n-2} /((n-2) / 2), 4 \pi_{n-1} / n$, \\
& $4 \pi_{n} / n$ \\
\hline$D_{n}$ for $n / 2 \notin \mathbb{Z}$ & $\pi_{1}, \pi_{2}, \pi_{3} / 2, \pi_{4} / 2, \ldots, \pi_{n-3} /((n-3) / 2), \pi_{n-2} /((n-1) / 2)$, \\
& $2 \pi_{n-1} /(n+3), 2 \pi_{n} /(n+3), 2\left(\pi_{1}+2 \pi_{n-1}\right) /(n+3), 2\left(\pi_{3}+2 \pi_{n-1}\right) /(n+5)$, \\
& $\ldots, 2\left(\pi_{n-2}+2 \pi_{n-1}\right) /(2 n), 2\left(\pi_{1}+2 \pi_{n}\right) /(n+3), 2\left(\pi_{3}+2 \pi_{n}\right) /(n+5)$, \\
\hline$G_{2}, 2\left(\pi_{n-2}+2 \pi_{n}\right) /(2 n)$ \\
\hline$F_{4}$ & $\pi_{1}, \pi_{2}$ \\
\hline$E_{7}$ & $\pi_{1}, \pi_{2} / 2, \pi_{3} / 3, \pi_{4}$ \\
\hline$E_{8}$ & $2 \pi_{1} / 3, \pi_{2} / 2,2 \pi_{3} / 7, \pi_{4} / 4, \pi_{5} / 3, \pi_{6}, 4 \pi_{7} / 5$ \\
\hline & $\pi_{1}, \pi_{2} / 3, \pi_{3} / 4, \pi_{4} / 6, \pi_{5} / 7, \pi_{6} / 5, \pi_{7} / 2, \pi_{8} / 4$ \\
\hline
\end{tabular}

may be nonconvex, which means that knowledge of the inner and outer boundaries is insufficient to describe it completely. Let $G: V$ be an irreducible representation. If the set of its weights contains the highest weight $\delta$ of the adjoint representation, we obtain a description of the inner boundaries of the Brion polytopes for the nullcone and for all of its irreducible components. If, moreover, the indices are sufficiently large, we can describe the Brion polytope itself for the nullcone.

Sjamaar, using the method of supports (see [17]), obtained a description of Bri( $V$ ) for representations all of whose indices differ from unity. By means of the method of supports and the Grosshans construction, we have been able to obtain a stronger result, namely the description of $\operatorname{Bri}(V)$ for almost all representations the set of whose indices does not contain 0 and 1 connected by an edge in the Dynkin diagram (for cases with branching Dynkin diagram a small additional restriction must be imposed). In particular, this class contains all representations with strictly dominant highest weights.

The topic of this paper was introduced to me by V. L. Popov, to whom I am much indebted for this as well as for his advice at the early stages of my work on this problem. I am most grateful also to my supervisor E. B. Vinberg for his critical reading of more than one version of this paper.

\section{List of notation:}

$G$ - a connected simply connected semisimple complex Lie group;

$T \subset G-$ a maximal torus of $G$;

$\mathcal{W}$ - the Weyl group of the pair $(G, T)$;

$B \subset G-$ a Borel subgroup of $G$, containing $T$;

$U \subset B-$ a maximal unipotent subgroup of $G$;

$K \subset G-$ a compact real form of $G$, such that $K \cap T$ is a maximal torus in $K$; 
$\mathfrak{g}, \mathfrak{t}, \mathfrak{k}$ - Lie algebras of $G, T, K$ respectively;

$P^{u}$ - the unipotent radical of the group $P$;

$\mathfrak{p}^{u}$ - the unipotent radical of the algebra $\mathfrak{p}$;

$V$ - an irreducible representation of $G$;

$(\cdot, \cdot)-K$-invariant Hermitian scalar product on $V$;

$\mu: P(X) \rightarrow \mathfrak{k}^{*}-$ moment map;

$\mathbb{E}$ - the space dual to the real form of $\mathfrak{t}\left(\mathbb{E}=i \operatorname{Lie}(T \cap K)^{*}\right)$;

$X(T) \subset \mathbb{E}$ - the set of characters of the torus $T$;

$\mathcal{C} \subset \mathbb{E}$ - the dominant Weyl chamber;

$\Delta$ - root system of $\mathfrak{g}$;

$\Delta^{+} \subset \Delta-$ the set of positive roots;

$\Pi$ - the system of simple roots;

$\Phi(V) \subset \mathbb{E}$ - the set of weights of $V$;

$M(V)=\operatorname{conv} \Phi(V)$ - the polytope of weights of $V$;

$X \subset V-$ an irreducible closed $G$-invariant cone;

$\left\{\pi_{1}, \ldots, \pi_{n}\right\}$ - the system of fundamental weights of $\mathfrak{g}$ (see [1]);

$e_{\alpha}$ - the root vector corresponding to the root $\alpha$.

\section{THE MOMENT MAP}

In the paper of Brion [7] it is proved that

$$
i \mu(P(X)) \cap \mathcal{C}=\operatorname{Bri}(X) .
$$

Thus our interest is in the intersection $i \mu(P(X)) \cap \mathbb{E}$.

Proposition 2.1 (Sjamaar [17]). The following condition holds:

$$
i \mu(\langle v\rangle) \in \mathbb{E} \Leftrightarrow\left(e_{\alpha} v, v\right)=0 \quad \forall \alpha \in \Delta .
$$

Let $V=\bigoplus_{\phi \in \Phi(V)} V_{\phi}$ be the weight decomposition of $V$, let $v \in V$ and let

$$
v=\sum_{\phi \in \Phi(V)} v_{\phi}, \quad v_{\phi} \in V_{\phi}
$$

be the weight decomposition of $v$. Let $\Phi(v)=\left\{\phi \mid v_{\phi} \neq 0\right\}$ be the set of weights of $v$. We call the convex hull of this set the support of $v$ and denote it by $\operatorname{supp} v$ (see [2]). We denote by $\langle v\rangle$ the point of the projective space corresponding to the vector $v$.

Definition 1. We say that $v$ has no adjacent weights if the difference of any two elements of $\Phi(v)$ is not a root.

Lemma 1. If $v \in X$ is a vector with no adjacent weights, and $X$ is the cone on $V$, then $\operatorname{supp} v \cap \mathcal{C} \subset \operatorname{Bri}(X)$.

Proof. It follows from Proposition 2.1 that if $v$ has no adjacent weights then $i \mu(\langle v\rangle) \in \mathbb{E}$. Consider the manifold $X^{\prime}:=\overline{T\langle v\rangle}$. Note that any vector $v^{\prime} \in X^{\prime}$ has no adjacent weights.

Now consider the moment map construction defined above, taking the large group as $T$, and $K \cap T$ in place of $K$. At the points satisfying $i \mu(\langle v\rangle) \in \mathbb{E}$, which include the whole manifold $X^{\prime}$, these two moment maps relative to the whole group and to the torus coincide. Hence to study the set $i \mu\left(X^{\prime}\right)=i \mu\left(X^{\prime}\right) \cap \mathbb{E}$ we may restrict ourselves to the moment map with respect to the torus. The set $X^{\prime}$ is an irreducible $T$-invariant manifold, and so its image under the moment map is a convex polytope (see for example [5]). 
Now let $A$ be a vertex of the support of the vector $v$. As can easily be shown, $\left\langle v_{A}\right\rangle \in \overline{T\langle v\rangle}$. We have $i \mu\left(\left\langle v_{A}\right\rangle\right)=A$, after which the convexity implies $\operatorname{supp} v=i \mu(\overline{T\langle v\rangle})$. Therefore

$$
\operatorname{Bri}(X) \supset \operatorname{Bri}\left(X^{\prime}\right) \cap \mathcal{C}=i \mu(\overline{T\langle v\rangle}) \cap \mathcal{C}=\operatorname{supp} c \cap \mathcal{C} .
$$

This lemma enables us to construct points of the Brion polytope. Note now that for any set of weights $Y \subset \Phi(V)$ we can construct a vector $v$ such that $\Phi(v)=Y$. Moreover, if this set is bounded away from zero, that is, $\exists \chi \in X(T):(m, \chi)>0 \quad \forall m \in Y$, then such a vector will lie in the nullcone (see [2], for example). Thus we have proved the following proposition.

Proposition 2.2. Let $Y \subset \Phi(V)$ be a set such that

$$
m_{1}-m_{2} \notin \Delta \quad \forall m_{1}, m_{2} \in Y .
$$

Then $\operatorname{conv}(Y) \cap \mathcal{C} \subset \operatorname{Bri}(V)$. Moreover, if

$$
\exists \chi \in X(T):(m, \chi)>0 \quad \forall m \in Y,
$$

then $\operatorname{conv}(Y) \cap \mathcal{C} \subset \operatorname{Bri}(\mathcal{N}(V))$.

This implies the following result.

Proposition 2.3 (Sjamaar, [17]). Let $G: V$ be an irreducible representation whose highest weight has no indices equal to 1 . Then

$$
\operatorname{Bri}(V)=M(V) \cap \mathcal{C} .
$$

In fact the set $Y=\mathcal{W} \xi$, where $\xi$ is the highest weight of the representation, satisfies the condition of the previous lemma, and its convex hull coincides with the polytope of weights of the representation.

\section{The local Structure theorem and a Description of $\operatorname{Bri}(V)$ FOR THE ADJOINT REPRESENTATION}

Let $G: V$ be an arbitrary finite-dimensional representation. As noted earlier, the outer boundaries of $\operatorname{Bri}(\mathcal{N}(V))$ and $\operatorname{Bri}(V)$ coincide, and therefore we shall work with $\operatorname{Bri}(V)$. We consider $U_{-}$-invariant functions on $V$, where $U_{-}$is the unipotent subgroup opposite to $U$. Then the definition of the Brion polytope can be reexpressed as

$$
\begin{array}{r}
\operatorname{Bri}(V)=\left\{-\lambda / n \mid \lambda \text { is a weight of a } U_{-} \text {-invariant homogeneous function on } V,\right. \\
\text { and } n \text { is its degree }\} .
\end{array}
$$

We start with a construction derived independently by Grosshans (see[11]) and Brion, Luna and Vust (see [8]). Let $v$ be any highest vector of $V$. Let $P$ be the stabilizer of $\langle v\rangle$, let $P_{-}$be the opposite parabolic subgroup, and let $L$ be a Levi subgroup common to both. The corresponding Lie algebras will be denoted by $\mathfrak{p}, \mathfrak{p}_{-}$and $\mathfrak{l}$. Let $V^{\prime}$ be an $L$-invariant complement to the line $\langle v\rangle$ in $V$, let $W^{\prime}$ be an $L$-invariant complement to $\mathfrak{p}_{-} v$ in $V$, and let $W=W^{\prime} \oplus\langle v\rangle$. Let $\hat{V}=V \backslash V^{\prime}$ and $\hat{W}=W \backslash W^{\prime}$. Then we have the following theorem.

Theorem 1 (Grosshans [11]; Brion-Luna-Vust [8]).

1) $\hat{W}$ is an L-invariant subset of $W$;

2) $\hat{V}$ is a $P_{-}$-invariant subset of $V$;

3) the morphism of the action $G \times V \rightarrow V$ induces a $P_{-}$-equivariant isomorphism $P_{-} *_{L} \hat{W} \rightarrow \hat{V}$ (see [2] regarding this notation). 


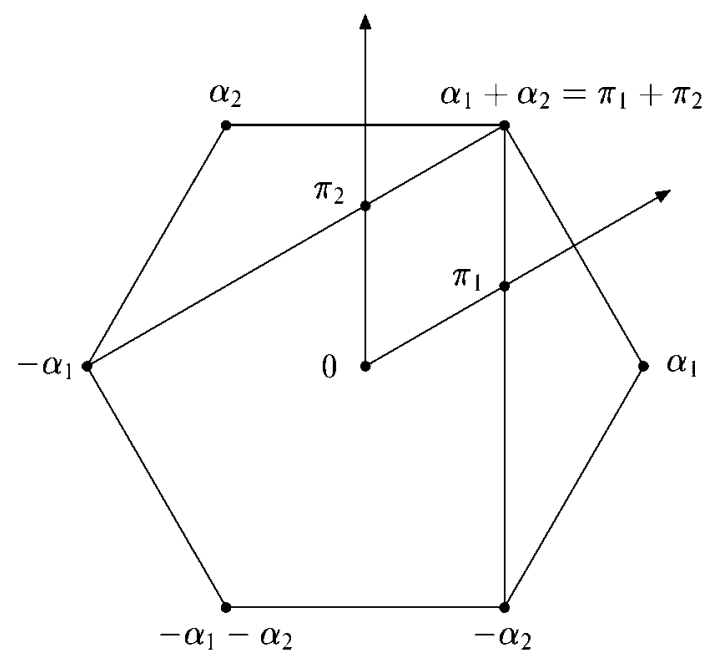

FIGURE 1. Brion polytope for the adjoint representation of $A_{2}$.

This fairly simple theorem is our main tool in many cases. The third statement implies an isomorphism

$$
\mathbb{C}[\hat{V}]^{U_{-}} \rightarrow \mathbb{C}[\hat{W}]^{U_{-} \cap L}
$$

of algebras of invariants. Unfortunately we cannot replace $\hat{V}$ by $V$ and $\hat{W}$ by $W$ in this formula. It is easy to see, however, that under this map the order of a zero of a function on the hyperplane complementary to $\langle v\rangle$ can only increase. Therefore we do have an embedding

$$
\mathbb{C}[V]^{U_{-}} \hookrightarrow \mathbb{C}[W]^{U_{-} \cap L}
$$

obtained by restricting the functions. A more detailed analysis of this map enabled Brion to prove a theorem on the local structure of the Brion polytope (see [7]). In the general case it cannot be applied to concrete calculations, but applying it in the neighborhood of the highest weight gives the following result.

Theorem 2 (Sjamaar, [17]). Let $V$ be an irreducible representation with highest weight $\lambda$, and let $\Phi(V)^{\prime}$ be the set of weights of $V$ that are not of the form $\lambda-\alpha$ where $\alpha$ is a root. Then $\operatorname{Bri}(V) \subset \mathcal{C} \cap M(V)^{\prime}$, where $M(V)^{\prime}:=\operatorname{conv} \Phi(V)^{\prime}$.

Example 3.1. In the case of the adjoint representation of the algebra $A_{2}$ the polytope of weights of $W$ turns out to be spanned by the weights $-\alpha_{1},-\alpha_{2},-\alpha_{1}-\alpha_{2}, \alpha_{1}+$ $\alpha_{2}$, so that the only edges that intersect the Weyl chamber are $\left[-\alpha_{1}, \alpha_{1}+\alpha_{2}\right]$ and $\left[-\alpha_{2}, \alpha_{1}+\alpha_{2}\right]$. Moreover, the ends of these edges do not differ by a root, and so we can apply Proposition 2.2 Hence the outer boundary of the Brion polytope of the adjoint representation for $A_{2}$ is $\left[\pi_{1}, \pi_{1}+\pi_{2}\right] \cup\left[\pi_{2}, \pi_{1}+\pi_{2}\right]$.

Note that in order to prove Sjamaar's theorem it is in fact not necessary to use Brion's local structure theorem: it suffices to use the embedding (3.1). Moreover, applying Brion's theorem only obstructs us in this case, since it restricts our knowledge of the polytope structure to a neighborhood of the highest weight. Using (3.1), however, we can repeat the process, taking $W$ as the new space and $L$ as the new group, and so on. This process is called constructing a Kostant cascade. Applying this technique we can obtain an outer bound, and this together with the moment map method allows us to 
describe the Brion polytope for many adjoint representations. To begin with, however, we note one obvious proposition:

Proposition 3.1. Let $Y \subset \mathbb{E}$ be a convex polytope with the origin in its interior, and let $\Gamma$ be a face of it that intersects every edge of the Weyl chamber. Then no other face of $Y$ intersects the interior of the Weyl chamber.

Theorem 3. For the adjoint representation of a simple algebra other than $E_{6}$ and $A_{n \geq 4}$, the outer boundary of the Brion polytope has the structure described in Table 1 .

Proof. The proof goes as follows: we shall construct a Kostant cascade, thereby obtaining outer bounds on the Brion polytope, and then bounding sets will be constructed in the image of the moment map. We use the notation $\varepsilon_{i}$ for weights, as in [1].

The case of $A_{1}$ is obvious, the case of $A_{2}$ was worked out in the example, and $A_{3}=D_{3}$.

Let $G=C_{n}$. In this case we do not even need to apply Theorem 1, since the highest weight has no index 1. By Theorem 2] $\operatorname{Bri}(V)=M(V) \cap \mathcal{C}$. We note that the outer boundary of the polytope is obtained as the intersection of the plane conv $\left\{2 \varepsilon_{1}, \ldots, 2 \varepsilon_{n}\right\}$ and the Weyl chamber.

Let $G=B_{n}$. The highest weight is $\lambda=\varepsilon_{1}+\varepsilon_{2}$. The algebra $\mathfrak{l}=A_{1} \oplus B_{n-1}$ is obtained by deleting the second vertex from the Dynkin diagram, and the space $W$ does not contain the weight components $V_{\varepsilon_{i} \pm \varepsilon_{j}}$ for $1 \leq i \leq 2$ and $j \geq 3$. We carry out the next step, taking $\lambda_{1}=\varepsilon_{3}+\varepsilon_{4}$. We then obtain that the algebra $\mathfrak{l}_{2}=A_{1} \oplus A_{1} \oplus A_{n-4}$ is obtained by deleting the second and fourth vertices from the Dynkin diagram, and $W_{2}$ does not contain the weight components $V_{\varepsilon_{i} \pm \varepsilon_{j}}$ for $3 \leq i \leq 4$ and $j \geq 5$. Proceeding in this way, we arrive at the fact that among the positive weights of $W_{k}$ there will be only $\varepsilon_{1} \pm \varepsilon_{2}, \varepsilon_{3} \pm \varepsilon_{4}, \ldots, \varepsilon_{n-1} \pm \varepsilon_{n}$ for even $n$, or $\varepsilon_{1} \pm \varepsilon_{2}, \varepsilon_{3} \pm \varepsilon_{4}, \ldots, \varepsilon_{n-2} \pm \varepsilon_{n-1}$, $\varepsilon_{n}$ for odd $n$. We denote these weights by $\phi_{1}, \ldots, \phi_{n}$. As is easily verified, none of the differences is equal to a root, and the subspace spanned by them is a face of the weight polytope of $V_{k}$, and moreover it intersects all edges of the Weyl chamber. Hence by Proposition 3.1, $\operatorname{Bri}(V) \subset \Phi\left(W_{k}\right) \cap \mathcal{C} \subset \operatorname{conv}\left(\phi_{i}, 0\right) \cap \mathcal{C}$, and by Proposition 1 we have $\operatorname{conv}\left(\phi_{i}, 0\right) \cap \mathcal{C} \subset \operatorname{Bri}(V)$. Hence we have described the outer boundary of the Brion polytope.

We carry out a similar procedure for $G_{2}$. Here $\lambda=3 \alpha_{1}+2 \alpha_{2}$. Among the weights of $W$ we do not find $3 \alpha_{1}+\alpha_{2}$. Therefore $\operatorname{Bri}(V) \subset \operatorname{conv}\left(0, \alpha_{1}, 3 \alpha_{1}+2 \alpha_{2}\right)$. It remains only to remark that the difference between the weights $\alpha_{1}$ and $3 \alpha_{1}+2 \alpha_{2}$ is not a root.

We carry out a similar procedure for $F_{4}$. We shall describe the highest weight, the algebra $\mathfrak{l}$, the vertices of the Dynkin diagram that are to be deleted, and the missing weights in $W$.

$\lambda=\varepsilon_{1}+\varepsilon_{2} . \mathfrak{l}_{1}=C_{3}$, there is no vertex number 3 , and $W_{1}$ does not contain the weights $\frac{1}{2}\left(\varepsilon_{1}+\varepsilon_{2} \pm \varepsilon_{3} \pm \varepsilon_{4}\right), \varepsilon_{i}$ and $\varepsilon_{i} \pm \varepsilon_{j}$, where $1 \leq i \leq 2$ and $3 \leq j \leq 4$.

$\lambda_{1}=\varepsilon_{1}-\varepsilon_{2} . \mathfrak{l}_{1}=C_{2}$, there is no vertex number 1 , and $W_{2}$ does not contain the weight $\frac{1}{2}\left(\varepsilon_{1}-\varepsilon_{2} \pm \varepsilon_{3} \pm \varepsilon_{4}\right)$.

$\lambda_{2}=\varepsilon_{3}+\varepsilon_{4} \cdot \mathfrak{l}_{1}=A_{1}$, there is no vertex number 2 , and $W_{3}$ does not contain the weights $\varepsilon_{3}$ and $\varepsilon_{4}$.

In $W_{3}$ the positive weights remaining are $\varepsilon_{1} \pm \varepsilon_{2}$ and $\varepsilon_{3} \pm \varepsilon_{4}$, and they satisfy the same conditions as the weights $\phi_{i}$ for $B_{n}$. The argument continues analogously.

We carry out a similar procedure for $E_{7}$. We shall describe the highest weight, the algebra $\mathfrak{l}$, the vertices of the Dynkin diagram that are to be deleted, and the missing weights in $W$.

$\lambda=-\varepsilon_{7}+\varepsilon_{8} \cdot \mathfrak{l}_{1}=D_{6}$, there is no vertex number 6 , and $W_{1}$ does not contain weights $-\varepsilon_{i}+\varepsilon_{8}, \varepsilon_{i}-\varepsilon_{7}$ and $\varepsilon_{l}+\varepsilon_{k}+\varepsilon_{s}+\varepsilon_{8}$, where $i, k, l, s<7$. 
$\lambda_{1}=\varepsilon_{1}+\varepsilon_{2}+\varepsilon_{7}+\varepsilon_{8} \cdot \mathfrak{l}_{2}=A_{1} \oplus D_{4}$, there is no vertex number 2 , and $W_{2}$ does not contain weights $\varepsilon_{1}-\varepsilon_{i}, \varepsilon_{2}-\varepsilon_{i}$ and $\varepsilon_{l}+\varepsilon_{k}+\varepsilon_{7}+\varepsilon_{8}$, where $2<i, k<7$ and $1 \leq l \leq 2$.

$\lambda_{2}=\varepsilon_{3}+\varepsilon_{4}+\varepsilon_{7}+\varepsilon_{8} \cdot \mathfrak{l}_{2}=A_{1} \oplus A_{1} \oplus A_{1} \oplus A_{1}$, there is no vertex number 4 , and $W_{3}$ does not contain weights $\varepsilon_{3}-\varepsilon_{i}, \varepsilon_{4}-\varepsilon_{i}$ and $\varepsilon_{l}+\varepsilon_{k}+\varepsilon_{7}+\varepsilon_{8}$, where $4<i, k<7$ and $3 \leq l \leq 4$.

In $W_{3}$ the only positive weights remaining are $\varepsilon_{1}-\varepsilon_{2}, \varepsilon_{3}-\varepsilon_{4}, \varepsilon_{5}-\varepsilon_{6},-\varepsilon_{7}+\varepsilon_{8}$, $\varepsilon_{1}+\varepsilon_{2}+\varepsilon_{7}+\varepsilon_{8}, \varepsilon_{3}+\varepsilon_{4}+\varepsilon_{7}+\varepsilon_{8}$ and $\varepsilon_{5}+\varepsilon_{6}+\varepsilon_{7}+\varepsilon_{8}$, and they satisfy the same conditions as the weights $\phi_{i}$ for $B_{n}$. The argument continues analogously.

We carry out a similar procedure for $E_{8}$. We shall describe the highest weight, the algebra $\mathfrak{l}$, the vertices of the Dynkin diagram that are to be deleted, and the missing weights in $W$.

$\lambda=\varepsilon_{1}-\varepsilon_{9} \cdot \mathfrak{l}_{1}=E_{7}$, there is no vertex number 1 , and $W_{1}$ does not contain weights $\varepsilon_{1}-\varepsilon_{i}, \varepsilon_{i}-\varepsilon_{9},-\varepsilon_{i}-\varepsilon_{j}-\varepsilon_{9}$ and $\varepsilon_{1}+\varepsilon_{i}+\varepsilon_{j}$, where $1<i, j<9$.

$\lambda_{1}=-\varepsilon_{1}-\varepsilon_{8}-\varepsilon_{9} . \mathfrak{l}_{2}=D_{6}$, there is no vertex number 6 , and $W_{2}$ does not contain weights $-\varepsilon_{1}-\varepsilon_{i}-\varepsilon_{9}, \varepsilon_{l}+\varepsilon_{k}+\varepsilon_{s}$ and $\varepsilon_{i}-\varepsilon_{8}$, where $1<l, k, s, i<9$.

$\lambda_{2}=\varepsilon_{2}+\varepsilon_{3}+\varepsilon_{8} . \mathfrak{l}_{3}=A_{1} \oplus D_{4}$, there is no vertex number 3 , and $W_{3}$ does not contain weights $\varepsilon_{i}-\varepsilon_{j}$ and $\varepsilon_{i}+\varepsilon_{j}+\varepsilon_{8}$, where $2 \leq i \leq 3$ and $3<j<8$.

$\lambda_{3}=\varepsilon_{4}+\varepsilon_{5}+\varepsilon_{8} . l_{4}=A_{1} \oplus A_{1} \oplus A_{1} \oplus A_{1}$, there is no vertex number 5 , and $W_{4}$ does not contain weights $\varepsilon_{i}-\varepsilon_{j}$ and $\varepsilon_{i}+\varepsilon_{j}+\varepsilon_{8}$, where $3 \leq i \leq 4$ and $4<j<8$.

In $W_{3}$ the only positive weights remaining are $\varepsilon_{2}-\varepsilon_{3}, \varepsilon_{4}-\varepsilon_{5}, \varepsilon_{6}-\varepsilon_{7}, \varepsilon_{1}-\varepsilon_{9}$, $-\varepsilon_{1}-\varepsilon_{8}-\varepsilon_{9}, \varepsilon_{2}+\varepsilon_{3}+\varepsilon_{8}, \varepsilon_{4}+\varepsilon_{5}+\varepsilon_{8}$ and $\varepsilon_{6}+\varepsilon_{7}+\varepsilon_{8}$, and they satisfy the same conditions as the weights $\phi_{i}$ for $B_{n}$. The argument continues analogously.

We carry out a similar procedure for $D_{n}$. We shall describe the highest weight, the algebra $\mathfrak{l}$, the vertices of the Dynkin diagram that are to be deleted, and the missing weights in $W$.

$\lambda=\varepsilon_{1}+\varepsilon_{2} . \mathfrak{l}_{1}=A_{1} \oplus D_{n-2}$, there is no vertex number 2 , and $W_{1}$ does not contain weights $\varepsilon_{i} \pm \varepsilon_{j}$, where $1 \leq i \leq 2$ and $3 \leq j \leq n$.

$\lambda_{1}=\varepsilon_{3}+\varepsilon_{4} \cdot \mathfrak{l}_{1}=A_{1} \oplus A_{1} \oplus D_{n-2}$, there is no vertex number 2 , and $W_{2}$ does not contain weights $\varepsilon_{i} \pm \varepsilon_{j}$, where $3 \leq i \leq 4$ and $3 \leq j \leq n$.

Continuing in this way, we arrive at two situations. Let $n$ be even. Then we obtain that in $W_{(n-3) / 2}$ the only positive roots remaining are $\varepsilon_{1} \pm \varepsilon_{2}, \varepsilon_{3} \pm \varepsilon_{4}, \ldots, \varepsilon_{n-1} \pm \varepsilon_{n}$, and they satisfy the same conditions as the weights $\phi_{i}$ for $B_{n}$. The argument continues analogously.

Now let $n$ be odd. Then we reach the situation

$$
\mathfrak{l}_{(n-3) / 2}=((n-3) / 2) A_{1} \oplus A_{3}
$$

and to this algebra there correspond all odd vertices and also vertex number $n-1$;

$\Phi\left(W_{(n-3) / 2}\right)=\Delta_{-} \cup\left\{\varepsilon_{1} \pm \varepsilon_{2}, \varepsilon_{3} \pm \varepsilon_{4}, \ldots, \varepsilon_{n-4} \pm \varepsilon_{n-3}\right\} \cup\left\{\varepsilon_{n-2} \pm \varepsilon_{n-1}, \varepsilon_{n-2} \pm \varepsilon_{n}, \varepsilon_{n-1} \pm \varepsilon_{n}\right\}$.

We further take $\lambda_{(n-3) / 2}=\varepsilon_{n-1}+\varepsilon_{n}$. Then $\mathfrak{l}_{(n-1) / 2}=((n-1) / 2) A_{1}$, and to this algebra there correspond all the odd vertices except the last; we have

$$
\Phi\left(W_{(n-1) / 2}\right)=\Delta_{-} \cup\left\{\varepsilon_{1} \pm \varepsilon_{2}, \varepsilon_{3} \pm \varepsilon_{4}, \ldots, \varepsilon_{n-2} \pm \varepsilon_{n-1}\right\} .
$$

After this we proceed in two ways. Taking the weights $\lambda_{(n-1) / 2}, \ldots, \lambda_{n-1}$ equal to $-\varepsilon_{2}+\varepsilon_{n}, \ldots,-\varepsilon_{n-2}+\varepsilon_{n}$ respectively, we obtain that

$$
\begin{aligned}
& \operatorname{Bri}(V) \subset \operatorname{conv}\left(\Delta_{-}\right. \cup\left\{\varepsilon_{1} \pm \varepsilon_{2}, \varepsilon_{3} \pm \varepsilon_{4}, \ldots, \varepsilon_{n-2} \pm \varepsilon_{n-1}\right\} \\
&\left.\backslash\left\{-\varepsilon_{1}+\varepsilon_{n},-\varepsilon_{3}+\varepsilon_{n}, \ldots,-\varepsilon_{n-2}+\varepsilon_{n}\right\}\right) \cap \mathcal{C} .
\end{aligned}
$$


Similarly, we also obtain

$$
\begin{aligned}
& \operatorname{Bri}(V) \subset \operatorname{conv}\left(\Delta_{-}\right. \cup\left\{\varepsilon_{1} \pm \varepsilon_{2}, \varepsilon_{3} \pm \varepsilon_{4}, \ldots, \varepsilon_{n-2} \pm \varepsilon_{n-1}\right\} \\
&\left.\backslash\left\{-\varepsilon_{1}-\varepsilon_{n},-\varepsilon_{3}-\varepsilon_{n}, \ldots,-\varepsilon_{n-2}-\varepsilon_{n}\right\}\right) \cap \mathcal{C} .
\end{aligned}
$$

We split the Weyl chamber into two parts $\mathcal{C}_{1}$ and $\mathcal{C}_{2}$ by the hyperplane $x_{1}=0$. Then

$$
\begin{aligned}
& \operatorname{Bri}(V) \subset \operatorname{conv}\left(\Delta_{-}\right. \cup\left\{\varepsilon_{1} \pm \varepsilon_{2}, \varepsilon_{3} \pm \varepsilon_{4}, \ldots, \varepsilon_{n-2} \pm \varepsilon_{n-1}\right\} \\
&\left.\backslash\left\{-\varepsilon_{1}+\varepsilon_{n},-\varepsilon_{3}+\varepsilon_{n} \ldots,-\varepsilon_{n-2}+\varepsilon_{n}\right\}\right) \cap \mathcal{C}_{1} \\
& \cup \operatorname{conv}\left(\Delta_{-} \cup\left\{\varepsilon_{1} \pm \varepsilon_{2}, \varepsilon_{3} \pm \varepsilon_{4}, \ldots, \varepsilon_{n-2} \pm \varepsilon_{n-1}\right\}\right. \\
&\left.\backslash\left\{-\varepsilon_{1}-\varepsilon_{n},-\varepsilon_{3}-\varepsilon_{n} \ldots,-\varepsilon_{n-2}-\varepsilon_{n}\right\}\right) \cap \mathcal{C}_{2} .
\end{aligned}
$$

Consider two hyperplanes: one given by $x_{1}+x_{3}+\cdots+x_{n-2}+x_{n}=1$ and the other by $x_{2}+x_{4}+\cdots+x_{n-1}+2 x_{n}=1$. It is easy to verify that these are both hyperplanes of faces $\Gamma_{1}$ and $\Gamma_{2}$ of the polytope $\Psi\left(W_{n}\right)$. Moreover, these faces together intersect the cone $\mathcal{C}_{1}$. We deal with the second half of the Weyl chamber similarly. We obtain the description as given in Table 1 and a simple verification shows that all these vertices lie in the image of the moment map.

Note that so far we have not been able to describe the polytope in the cases of $A_{\geq 4}$ and $E_{6}$, with the greatest difficulties arising in the case of $D_{n}$ for $n$ odd. What is it that characterizes these cases? Consider the set of weights $\lambda$ for which the space $V_{(\lambda)}$ is selfadjoint. This forms a lattice. Let $b$ be its codimension in the weight lattice. In the cases when $b=0$, that is, when all representations are selfadjoint, we obtain the following a posteriori result: the outer boundary of the Brion polytope is the intersection of the Weyl chamber with the subspace spanned by $n$ nonadjacent weights, with this subspace completely covering the Weyl chamber. In cases when $b=1$, that is, $A_{2}$ and $D_{2 n+1}$, the complexity of both the proof and the answer increases sharply. In the cases when $b>1$ we have been unable to obtain a conclusive result. Thus it is clear that the problems are connected with the presence of nonselfadjoint representations. The structure of this connection is not clear, but we are able to proceed as follows. We introduce the notion of a selfadjoint Brion polytope:

$$
C(X)_{s}=\left\{x \in X(T) \otimes_{\mathbb{Z}} \mathbb{Q} \mid \exists n \in \mathbb{Z}_{\geq 0}, \lambda \in \chi(T): x=\lambda / n, m_{\lambda^{*}, n} \neq 0, \lambda=\lambda^{*}\right\}
$$

and $\operatorname{Bri}(X)_{s}=\operatorname{conv}\left(C(X)_{s}\right)$. It is easy to show that this set is the intersection of the Brion polytope with the space $\mathbb{E}_{s}$ spanned by the weights of the selfadjoint representations. We prove (a posteriori) the following theorem:

Theorem 4. The outer boundary of a selfadjoint Brion polytope for the adjoint representation is the intersection of the space $\mathbb{E}_{s}$, the Weyl chamber, and the space spanned by $n-b$ nonadjacent self-dual weights, with this space completely covering the intersection of the Weyl chamber and the space $\mathbb{E}_{s}$, that is, it intersects every ray from the origin lying in this cone.

Proof. For the case $b=0$ everything has already been proved, and for $b=1$ it is easy to obtain the description by intersecting $C(V)$ and $\mathbb{E}_{s}$. Let now $b>1$, that is, $\mathfrak{g}=A_{\geq 4}$ or $\mathfrak{g}=E_{6}$.

Let $\mathfrak{g}=A_{n}$. Then:

$\lambda=\varepsilon_{1}+\varepsilon_{n} . \mathfrak{l}_{1}=A_{n-2}$, there is no vertex number 1 or $n$, and $W_{1}$ does not contain weights $\varepsilon_{1}-\varepsilon_{i}$ and $\varepsilon_{i}-\varepsilon_{n}$, where $1<i<n$.

$\lambda_{1}=\varepsilon_{2}+\varepsilon_{n-1} \cdot \mathfrak{l}_{2}=A_{n-4}$, there is no vertex number 2 or $n-1$, and $W_{2}$ does not contain weights $\varepsilon_{2}-\varepsilon_{i}$ and $\varepsilon_{i}-\varepsilon_{n-1}$, where $1<i<n-1$. 
Continuing in this way we obtain that the desired $n-b$ selfadjoint weights are $\varepsilon_{1}-\varepsilon_{n}$, $\varepsilon_{2}-\varepsilon_{n-1}, \ldots, \varepsilon_{[n / 2]}-\varepsilon_{] n / 2[+1}$.

In the case of $E_{6}$ after the first step the construction proceeds just as for $A_{5}$.

\section{Description of $\operatorname{Bri}(V)$ For most irreducible Representations}

Let the indices of the highest weight $\lambda$ be at least two. As Sjamaar's theorem tells us, the Brion polytope for the whole space is the intersection of the polytope of weights and the Weyl chamber, that is, the intersection of the two cones $\mathcal{C}$ and $\lambda-\operatorname{con} \Pi$. The vertices of this polytope can only be the intersections of $k$-dimensional faces of one cone with $(n-k)$-dimensional faces of the other. Let the face $\left\{\sum_{i \in I} t_{i} \pi_{i}\right\}$ with $t_{i} \geq 0$ intersect the face $\lambda-\left\{\sum_{j \in J} t_{j}^{\prime} \alpha_{j}\right\}$, where $t_{j}^{\prime} \geq 0$. Notice now that in the decomposition of a simple root into fundamental weights the only coefficient that can be positive is the one for the weight with the same number. By hypothesis, the highest weight $\lambda$ is strictly dominant, and so $I \cup J=\{1, \ldots, n\}$. Moreover, the sets $I$ and $J$ do not intersect, from dimensional considerations.

Let $\mathfrak{g}_{I}$ be the subalgebra corresponding to the subset $I$ of the Dynkin diagram. The fact that any faces corresponding to mutually complementary sets must intersect follows from the fact that for $\mathfrak{g}_{I}$ the dominant Weyl chamber lies in the cone spanned by the simple roots. Thus we have proved the following lemma:

Lemma 2. Let all the indices of the highest weight be at least two. Then $\operatorname{Bri}(V)$ has $2^{n}$ vertices, corresponding to the subsets of $\{1, \ldots, n\}$. To the subset $I \subset\{1, \ldots, n\}$ there corresponds the vertex $A_{I}$ defined by

$$
A_{I}=\sum_{i \in I} c_{i} \pi_{i}=\lambda-\sum_{i \notin I} c_{i}^{\prime} \alpha_{i} .
$$

Let $\Gamma_{I}$ be the face of the weight polytope having centroid $I$, and let $\mathfrak{g}_{I}: V_{I}$ be the subrepresentation corresponding to vectors whose weights lie in this face.

Proposition 4.1. The intersection of $\operatorname{Bri}(V)$ with the face $\Gamma_{I}$ of the polytope $M(V)$ coincides with $\operatorname{Bri}\left(V_{I}\right)$.

The proof of this proposition can be found in 6 . Now, in the case when the indices of the highest weights are at least two it is easy to obtain an alternative proof of Sjamaar's theorem that if the indices are different from unity then $\operatorname{Bri}(V)=M(V)^{\prime}$. Indeed, in this case any representation $\mathfrak{g}_{I}: V_{I}^{\prime}$ has nontrivial invariants (which can be checked from the lists in [3] and [4]), and hence $0 \in \operatorname{Bri}\left(V_{I}^{\prime}\right)$ and $A_{I} \in \operatorname{Bri}(V)$ for all $I$.

Now suppose there are no zero indices, but possibly some ones. A similar proof does not work, since for $I=\{1, \ldots, n\} \backslash\{i\}$ where $m_{i}=1$ we obtain $\mathfrak{g}_{I}: V_{I}^{\prime}=A_{1}: V_{\pi_{1}}$, and this representation has no nontrivial invariants. However, it is easy to see that for all other $I$ the representations $\mathfrak{g}_{1}: V_{i}^{\prime}$ do have invariants, and therefore if $I \neq\{1, \ldots, n\} \backslash\{i\}$ where $m_{i}=1$, then $A_{I} \in \operatorname{Bri}(V)$. Now let $S$ be the set of points lying on the same side of the space spanned by the set $L=\lambda-\Pi$ as does 0 . Applying Theorem 1 we obtain

$$
\operatorname{Bri}(V) \subset M(V)^{\prime}=M(V) \bigcap_{j \mid m_{j}=1} s_{\alpha_{j}}(S),
$$

where $s_{\alpha} \in W$ is the reflection in the hyperplane orthogonal to $\alpha$, and $\Phi(V)^{\prime}=\Phi(V) \backslash\{\lambda-$ $\Pi\}$. Each subspace $s_{\alpha_{j}}(\operatorname{conv}(L))$ intersects all edges

$$
\left[A_{\{1, \ldots, n\} \backslash\{i\}}, A_{\{1, \ldots, n\} \backslash\{i, j\}}\right]
$$

and contains the vertex $A_{1, \ldots, n}$. Moreover, by Lemma 2.2 applied to the set $s_{\alpha_{i}}(\lambda-\Pi)$, we obtain $\operatorname{Bri}(V) \supset s_{\alpha_{i}}(\operatorname{conv}(L)) \cap \mathcal{C}$. Thus we have proved the following theorem: 
Theorem 5. Let the highest weight be strictly dominant. Then the Brion polytope of the entire representation is equal to $M(V)^{\prime} \cap \mathcal{C}$. The vertices of this set are the points $A_{I}$ for $\# I \neq\{1, \ldots, n\} \backslash\{i\} \mid m_{i}=1$ and the points

$$
\left[A_{\{1, \ldots, n\} \backslash\{i\}}, A_{\{1, \ldots, n\} \backslash\{i, j\}}\right] \cap s_{\alpha_{i}}(\operatorname{conv}(L))
$$

for those $i$ with $m_{i}=1$.

Our aim will be to obtain an analogous description in the more general case. Suppose the highest weight is not strictly dominant. In this case the vertices of the intersection $M(V) \cap \mathcal{C}$ still lie in the set $\left\{A_{I}\right\}$, but this time some of the points $A_{I}$ will coincide. A more detailed investigation proves the following proposition:

Proposition 4.2. Let $K=\left\{i \mid m_{i}=0\right\}$, and $I \subset\{1, \ldots, n\}$. We split the roots with numbers from the set $\{1, \ldots, n\} \backslash I$ into those contained in connected components of the Dynkin diagram, and let $T$ be the set of vertices of the components lying entirely in the graph corresponding to $K$. Then for all $I^{\prime}$ with $I \subset I^{\prime} \subset I \cap T$ we have $A_{I}=A_{I^{\prime}}$.

Proposition 4.3. Let $K=\left\{i \mid m_{i}=0\right\}$, and $I \subset\{1, \ldots, n\}$. Let $T \subset I \cap K$ be the set of vertices not adjacent to the vertices $\{1, \ldots, n\} \backslash I$ by edges of the Dynkin diagram. Then for all $I^{\prime}$ with $I \backslash T \subset I^{\prime} \subset I$ we have $A_{I}=A_{I^{\prime}}$.

Proposition 4.4. If $A_{I}=A_{I^{\prime}}$, then $I$ and $I^{\prime}$ can be obtained from each other by a sequence of applications of the processes from the preceding two propositions.

Thus if all representations $\mathfrak{g}_{A}: V_{A}^{\prime}$ have nontrivial invariants, then all points $A_{I}$ lie in $\operatorname{Bri}(V)$, and the Brion polytope can be constructed extremely easily. However, this happens only in the case when $m_{i} \neq 1$ for all $i$, that is, just in the case worked out by Sjamaar. Note that our approach gives an alternative proof of this theorem. Unfortunately it turns out that if indices 0 and 1 are connected by an edge of the Dynkin diagram, then the local structure theorem in the general case does not give sufficient bounds (with some exceptions).

We now turn to the remaining cases. Suppose the Dynkin diagram has no indices 0 and 1 connected by an edge, and also another small additional condition is satisfied, namely for $E_{n}$ we have $m_{n-3} \neq 0$, and for $D_{n}$ if $m_{n-2}=0$ then also $m_{n-1}=m_{n}=0$. Then we are able to obtain a description of the Brion polytope.

We shall do this as follows. First note that all the points $A_{I}$ for $I \neq\{1, \ldots, n\} \backslash\{i\}$, where $m_{i}=0$, lie in the Brion polytope (here we use our basic condition on the indices). Thus the desired polytope is obtained by "deleting" the remaining vertices and certain neighborhoods of them. We fix some $j$ with $m_{j}=0$.

Consider the subgraph of the Dynkin diagram corresponding to the set of zero indices, and split it into its connected components, whose sets of vertices we denote by $M_{1}^{\prime}, \ldots, M_{k}^{\prime}$. We add to each such component the vertices adjacent to it (of which there are one or two; there cannot be three in view of the additional restriction given in the statement of the theorem). We denote the sets of vertices so obtained by $M_{1}, \ldots, M_{k}$. Our problem is to construct a system $\mathcal{S}$ of sets $S$ such that the condition $\operatorname{Bri}(V) \subset$ conv $(0, S)$ appears as a result of applying a Kostant cascade, and then to prove that the polytope so obtained lies in the image of the moment map. We shall construct these sets as the unions of sets $S_{i}$ for all $i \in\{i, \ldots, k\}$ and points $s_{j}\left(\lambda-\alpha_{i}\right)$ for all $i$ such that $m_{i}>0$. The problem reduces to the analogous one for the spaces $s_{\alpha}\left(V_{M_{i}}\right)$ where $V_{M}$ is the space obtained as the linear span of the weight subspaces of $V$ with weights lying in the face $\lambda-\sum_{i \in M} c_{i} \alpha_{i}$. Indeed, after the first step of applying Theorem $\square$ the algebra $\mathfrak{l}$ splits into the direct sum of simple components corresponding to the sets $M_{i}^{\prime}$, and $s_{\alpha}\left(V_{M_{i}}\right)$ is stabilized by all these components apart from the component with the 
same number. Furthermore, since zero indices on the Dynkin diagram are not adjacent to those with index 1 , we can apply the reflection $s_{\alpha}$ and solve the following problem.

Let $\mathfrak{g}_{i}$ be a simple Lie algebra corresponding to a Dynkin diagram without branching, and let $V_{i}$ be a representation whose highest weight $\lambda_{i}$ can have nonzero indices only on the ends of the diagram, or $\mathfrak{g}_{i}=D_{n}$ and only $m_{1} \neq 0$. Next let $V_{i}^{\prime}$ be a $T$-invariant complement to $\mathbb{C} v_{\left(\lambda^{\prime}\right)}$ in $V_{i}$, and let $\mathfrak{g}_{i}^{\prime}$ be the subalgebra corresponding to the zero indices. We now start applying Theorem 1 to this space and algebra. The problem is to construct systems $\mathcal{S}_{1}^{\prime}$ and $\mathcal{S}_{2}^{\prime}$ of sets such that the bound conv $\left(V_{i}^{\prime}\right) \subset \operatorname{conv}\left(0, S^{\prime}\right)$ for all $S \in \mathcal{S}_{1}^{\prime}$ is obtained by repeated application of this theorem, starting with $V_{i}^{\prime}$ and $\mathfrak{g}_{i}^{\prime}$, and such that in each set $S \in \mathcal{S}_{2}^{\prime}$ there are no adjacent weights and

$$
\bigcap_{S^{\prime} \in \mathcal{S}_{1}^{\prime}} \operatorname{conv}\left(0, S^{\prime}\right)=\operatorname{conv}_{S^{\prime} \in \mathcal{S}_{1}^{\prime}}\left(\mathcal{C} \cap \operatorname{conv}\left(S^{\prime}\right)\right) .
$$

Indeed, if we can construct these sets for the faces, we can apply reflection and then take the union.

Now we proceed to analyze the various cases. To begin, let $\mathfrak{g}_{i}=A_{k}, m_{2}=m_{3}=$ $\cdots=m_{k}=0, m_{1}>1$. Then from the point $\lambda_{i}$ there depart the edges with directions $\left\{\alpha_{1}+\alpha_{2}+\cdots+\alpha_{k}, \ldots, \alpha_{1}+\alpha_{2}, \alpha_{1}\right\}$. The nearest weights on these edges are the adjacent ones, and so we carry out the second step of Theorem 1 and obtain the bound:

$$
\operatorname{Bri}\left(V_{i}^{\prime}\right) \subset \operatorname{conv}\left(\lambda_{i}-2 \alpha_{1}-2 \alpha_{2}-\cdots-2 \alpha_{k}, \ldots, \lambda_{i}-2 \alpha_{1}-2 \alpha_{2}, \lambda_{i}-\alpha_{1}\right) .
$$

There are already no neighbors among these weights and so we obtain the desired set $S_{1}=S_{2}$.

The cases $\mathfrak{g}_{i}=B_{n}, \mathfrak{g}=C_{n}, \mathfrak{g}=D_{n}$ and $m_{2}=m_{3}=\cdots=m_{k}=0, m_{1}>1$ are analyzed similarly. The cases $\mathfrak{g}_{i}=B_{n}, \mathfrak{g}_{i}=C_{n}$ and $m_{1}=m_{2}=\cdots=m_{k-1}=0$, $m_{k}>1$ are possible only for the subalgebra of $F_{4}$ with $k=3$ and also do not give rise to any complications. Now we turn to the more complicated case when there are nonzero indices on both sides of the Dynkin diagram for $\mathfrak{g}_{i}$.

Let $\mathfrak{g}_{i}=A_{k}, m_{2}=m_{3}=\cdots=m_{k-1}=0, m_{1}>1, m_{k}>1$. Then from $\lambda_{i}$ there depart edges in the directions $\left\{\alpha_{2}+\cdots+\alpha_{k-1}+\alpha_{k}, \ldots, \alpha_{k-1}+\alpha_{k}, \alpha_{k}\right\}$ and $\left\{\alpha_{1}+\alpha_{2}+\right.$ $\left.\cdots+\alpha_{k-1}, \ldots, \alpha_{2}+\alpha_{1}, \alpha_{1}\right\}$.

Among the closest points on these edges there are quite a lot of neighbors. We can apply Theorem 1 to the highest weight $\lambda_{i}-\alpha_{1}$ and then to $\lambda_{i}-\alpha_{k}$. After this we obtain the bound

where

$$
\operatorname{Bri}\left(V_{i}^{\prime}\right) \subset \operatorname{conv}(0, L)
$$

$$
\begin{gathered}
L:=\left\{\lambda_{i}-\alpha_{1}, \lambda_{i}-2 \alpha_{1}-2 \alpha_{2}, \ldots, \lambda_{i}-2 \alpha_{1}-\cdots-2 \alpha_{k}, \lambda_{i}-\alpha_{2}-\cdots-\alpha_{k-1}-\alpha_{k},\right. \\
\left.\lambda_{i}-2 \alpha_{3}-\cdots-2 \alpha_{k-1}-2 \alpha_{k}, \ldots, \lambda_{i}-2 \alpha_{k-1}-2 \alpha_{k}, \lambda_{i}-\alpha_{k}\right\} .
\end{gathered}
$$

Unfortunately there are two neighbors among these weights, and therefore a simple application of Theorem [1 is not enough. We divide the Weyl chamber into two parts by the plane $x_{1}+x_{n}=m_{1}-m_{n}$, where $x_{i}$ are the coefficients of the decomposition of the vector by the $\varepsilon_{i}$ and $\sum_{i} x_{i}=0$. We shall prove that the intersection of the polytope

$P_{1}:=\operatorname{conv}\left(\Phi\left(V_{i}^{\prime}\right) \backslash\left(\lambda_{i}-\left\{\alpha_{1}+\alpha_{2}, \ldots, \alpha_{1}+\cdots+\alpha_{k-1}, \alpha_{3}+\cdots+\alpha_{k}, \ldots, \alpha_{k-1}+\alpha_{k}\right\}\right)\right)$ with the Weyl chamber and with the half-space $x_{1}+x_{n}=m_{1}-m_{n}$ is contained in $\operatorname{conv}_{S^{\prime} \in \mathcal{S}_{1}^{\prime}}\left(\mathcal{C} \cap \operatorname{conv}\left(S^{\prime}\right)\right)$. For this we study the outer faces of the polytope $P_{1}$. They can be divided into two types: subsets of faces of conv $\left(\Phi\left(V_{i}^{\prime}\right)\right)$ and faces spanned by points of $L$. The faces that interest us are those containing simultaneously the points $\lambda_{i}-\alpha_{k}$ and $\lambda_{i}-\alpha_{2}-\cdots-\alpha_{k}$. Any face $P_{1}$ of the first type is a subset of a face of conv $\left(\Phi\left(V_{i}^{\prime}\right)\right)$ containing the weight $\lambda_{i}$. Each of these lies in the cone with vertex at $\lambda_{i}$ and edges 
$-\varepsilon_{1}+\varepsilon_{2}$ or $-\varepsilon_{2}+\varepsilon_{n+1},-\varepsilon_{1}+\varepsilon_{3}$ or $-\varepsilon_{3}+\varepsilon_{n+1}, \ldots,-\varepsilon_{1}+\varepsilon_{n}$ or $-\varepsilon_{n}+\varepsilon_{n+1}$, with one element being chosen from each pair. If it contains $\lambda_{i}-\alpha_{k}$ and $\lambda_{i}-\alpha_{2}-\cdots-\alpha_{k}$, then it is easy to verify that it either does not intersect the Weyl chamber, or is obtained by choosing the last element from each pair. In this case, however, it lies completely in the other half-space and does not interest us.

Now we turn to the second type of face, i.e. those faces spanned by points of $L$. It is easy to check that any point of this set can be joined by an edge either to $\lambda_{i}-c\left(\alpha_{1}-\right.$ $\left.\cdots-\alpha_{j}\right)$, or to $\lambda_{i}-c\left(\alpha_{j+1}-\cdots-\alpha_{n}\right)$. Moreover, the points $\lambda_{j}-\alpha_{1}, \lambda_{i}-\alpha_{2}-\cdots-\alpha_{k}$ are joined by an edge. Thus the points $\lambda_{i}-\alpha_{1}$ and $\lambda_{i}-\alpha_{2}-\cdots-\alpha_{k}$ lie in any such face, and moreover, from any two $\lambda_{i}-2\left(\alpha_{1}-\cdots-\alpha_{i}\right)$ and $\lambda_{i}-2\left(\alpha_{j+1}-\cdots-\alpha_{n}\right)$ with $j>1$, exactly one lies in the face. If this face intersects the interior of the Weyl chamber and contains the point $\lambda_{j}-\alpha_{k}$ then this set has to comprise $\lambda_{i}-\alpha_{2}-\cdots-\alpha_{k} ; \lambda_{i}-\alpha_{1}$, $\lambda_{i}-\alpha_{1}-\alpha_{2}, \ldots, \lambda_{i}-\alpha_{1}-\alpha_{2}-\cdots-\alpha_{j}, \lambda_{i}-\alpha_{j+2}-\cdots-\alpha_{k}, \ldots, \lambda_{i}-\alpha_{k}$. We divide this simplex into two by breaking the edge $\left[\lambda_{i}-\alpha_{1}, \lambda_{i}-\alpha_{2}-\cdots-\alpha_{k}\right]$ at its point $A$ of intersection with the hyperplane. One of these simplices, namely the one not containing $\lambda_{i}-\alpha_{1}$, is either disjoint from the interior of the Weyl chamber (when $j>1$ ), or does not meet the half-space that we are interested in.

We now study the second of these two simplices. It is easy to verify that its intersection with the Weyl chamber has the structure of a pyramid with vertex at $A$. Therefore it lies entirely in $M_{2}$, since the point $A$ also lies there (the weights $\lambda_{i}-\alpha_{1}$ and $\lambda_{i}-\alpha_{2}-\cdots-\alpha_{k}$ are not adjacent), and the intersection of the side of the face opposite to $\lambda_{i}-\alpha_{2}-\cdots-\alpha_{k}$ also lies there (since if we remove this point then we no longer have any adjacent weights).

Thus we can describe the intersection of the desired polytope with the half-space. Its intersection with the other half-space is found in a similar way. We obtain the following polytope:

$$
\begin{aligned}
& \operatorname{conv}\left(0, \lambda_{i}-\alpha_{1}, \lambda_{i}-2 \alpha_{1}-2 \alpha_{2}, \ldots, \lambda_{i}-2 \alpha_{1}-\cdots-2 \alpha_{k-1},\right. \\
& \left.\lambda_{i}-2 \alpha_{2}-\cdots-2 \alpha_{k-1}-2 \alpha_{k}, \ldots, \lambda_{i}-2 \alpha_{k-1}-2 \alpha_{k}, \lambda_{i}-\alpha_{k}, \lambda_{i}-\frac{\alpha_{1}+\cdots+\alpha_{n}}{2}\right) .
\end{aligned}
$$

In the case of $C_{n}$ these difficulties do not arise, and the case of $B_{n}$ is analyzed similarly.

In order to formulate the theorem we proceed as follows.

For each space $V_{i}$ depending on its type we define the set $L_{i}$ accordingly:

- If $\mathfrak{g}_{i}=A_{k}$ or $\mathfrak{g}_{i}=C_{k}$ and only $m_{1}>0$ then

$$
L_{i}=\left\{\lambda_{i}-\alpha_{1} ; \lambda_{i}-2 \alpha_{1}-2 \alpha_{2}, \ldots, \lambda_{i}-2 \alpha_{1}-\cdots-2 \alpha_{k}\right\} \text {. }
$$

- If $\mathfrak{g}_{i}=B_{k}$ and only $m_{1}>0$ then

$L_{i}=\left\{\lambda_{i}-\alpha_{1} ; \lambda_{i}-2 \alpha_{1}-2 \alpha_{2}, \ldots, \lambda_{i}-2 \alpha_{1}-\cdots-2 \alpha_{k-1} ; \lambda_{i}-\alpha_{1}-\cdots-\alpha_{k}\right\}$.

- If $\mathfrak{g}_{i}=B_{3}$ and only $m_{3}>0$ then

$$
L_{i}=\left\{\lambda_{i}-\alpha_{3}, \lambda_{i}-2 \alpha_{3}-2 \alpha_{2}, \lambda_{i}-2 \alpha_{3}-2 \alpha_{2}-2 \alpha_{1}\right\} .
$$

- If $\mathfrak{g}_{i}=C_{3}$ and only $m_{3}>0$ then

$$
L_{i}=\left\{\lambda_{i}-\alpha_{3}, \lambda_{i}-\alpha_{3}-2 \alpha_{2}, \lambda_{i}-\alpha_{3}-2 \alpha_{2}-2 \alpha_{1}\right\} .
$$

- If $\mathfrak{g}_{i}=D_{k}$ and only $m_{1}>0$ then

$$
\begin{aligned}
& L_{i}=\left\{\lambda_{i}-\alpha_{1} ; \lambda_{i}-2 \alpha_{1}-2 \alpha_{2}, \ldots, \lambda_{i}-2 \alpha_{1}-\cdots-2 \alpha_{k-1}\right. \\
&\left.\lambda_{i}-\alpha_{1}-2 \alpha_{2}-\cdots-2 \alpha_{k-2}-\alpha_{k-1}-\alpha_{k}\right\} .
\end{aligned}
$$


- If $\mathfrak{g}_{i}=A_{k}$ or $\mathfrak{g}_{i}=B_{k}$ and both $m_{1}>0$ and $m_{k}>0$ then

$$
\begin{aligned}
& L_{i}=\left\{\lambda_{i}-\alpha_{1} ; \lambda_{i}-2 \alpha_{1}-2 \alpha_{2}, \ldots, \lambda_{i}-2 \alpha_{1}-\cdots-2 \alpha_{k-1} ;\right. \\
& \left.\quad \lambda_{i}-2 \alpha_{2}-\cdots-2 \alpha_{k-1}-2 \alpha_{k}, \ldots, \lambda_{i}-2 \alpha_{k-1}-2 \alpha_{k} ; \lambda_{i}-\alpha_{k} ; \lambda_{i}-\frac{\alpha_{1}+\cdots+\alpha_{n}}{2}\right\} .
\end{aligned}
$$

- If $\mathfrak{g}_{i}=C_{k}$ and both $m_{1}>0$ and $m_{k}>0$ then

$$
\begin{aligned}
L_{i}=\left\{\lambda_{i}-\alpha_{1}, \lambda_{i}-\alpha_{1}-2 \alpha_{2}, \ldots, \lambda_{i}-\alpha_{1}-2 \alpha_{2}-\cdots-2 \alpha_{k-1} ;\right. \\
\left.\lambda_{i}-2 \alpha_{2}-\cdots-2 \alpha_{k-1}-2 \alpha_{k}, \ldots, \lambda_{i}-2 \alpha_{k-1}-2 \alpha_{k} ; \lambda_{i}-\alpha_{k}\right\} .
\end{aligned}
$$

Theorem 6. Let the highest weight of the space $V$ satisfy the following conditions: there are no indices 0 and 1 connected by an edge, and moreover for $E_{n}$ we have $m_{n-3} \neq 0$ and for $D_{n}$ if $m_{n-2}=0$ then also $m_{n-1}=m_{n}=0$. Then the Brion polytope $\operatorname{Bri}(V)$ has the following structure.

Let $L=\left(\bigcup L_{i}\right) \cup\left\{\lambda-\alpha_{i} \mid m_{i}>1\right\}$. The outer boundary of the convex hull of $L$ cuts the polytope $M(V)$ into two parts. Denote the part that contains zero by $S$. Then

$$
\operatorname{Bri}(V)=\bigcap_{i, \text { where } m_{i}=1} s_{\alpha_{i}}(S) \cap M(V) \cap \mathcal{C} .
$$

The vertices of this polytope are the points $A_{I}$ for $I \neq\{1, \ldots, n\} \backslash\{i\}$, where $m_{i}=1$, and the points of intersection of the intervals $\left[A_{\{1, \ldots, n\} \backslash\{i\}}, A_{\{1, \ldots, n\} \backslash\{i, j\}}\right]$, where $m_{i}=1$, with the boundary of the set $s_{\alpha_{i}}(M)$.

\section{INNER BOUNDS AND A DESCRIPTION OF THE INNER BOUNDARY OF $\operatorname{Bri}(\mathcal{N}(V))$}

FOR REPRESENTATIONS WITH HIGHEST WEIGHT BELONGING TO THE ROOT LATTICE

We now take up the problem of describing the inner boundary of $\operatorname{Bri}(\mathcal{N}(V))$. We start by obtaining bounds on the Brion polytope. Let $x \in \operatorname{Bri}(\mathcal{N}(V))$. Then

$$
\exists n \in \mathbb{N}: V_{n x^{*}} \in \mathbb{C}[\mathcal{N}(V)]_{n} .
$$

Hence there exists a $U$-invariant homogeneous regular function $f$ of degree $n$ and of weight $(n x)^{*}$ (that is $\left.t f=(n x)^{*}(t) f \forall t \in T\right)$, not identically zero on the nullcone. This function has the form $f(v)=\psi\left(v^{\otimes n}\right)$, where $\psi$ is a $U$-invariant linear function of weight $(n x)^{*}$ on $S^{n} V$, and $V \rightarrow S^{n} V, v \rightarrow v^{\otimes n}$ is the Veronese map. Since $f$ is not identically zero on the nullcone, there is an orbit $\mathcal{O} \subset \mathcal{N}(V)$ such that $f$ is not identically zero on $\mathcal{O}$. Therefore $V_{n x} \subset S^{n} V$, and for any $v \in \mathcal{O}$ the projection $v^{\otimes n} \rightarrow V_{n x}$ is not equal to zero (because of the $G$-equivariance of the Veronese embedding).

We now choose a vector $v \in \mathcal{O}$ such that $0 \notin \operatorname{supp} v$, and the characteristic $h_{v}$ of $v$ is dominant (see [2]). Then the nontriviality of the projection implies

$$
n \operatorname{supp} v \cap \Phi\left(V_{n x}\right) \neq \emptyset,
$$

but then $\exists y \in \operatorname{supp} v: n y \in n x-\operatorname{con}(\Pi)$, where con $(Y)$ is the cone spanned by the set $Y$. This last condition may be reexpressed as $\exists y \in \operatorname{supp} v: n x \in n y+\operatorname{con}(\Pi)$ or as

$$
\operatorname{Bri}(\mathcal{N}(V)) \subset \operatorname{supp}(v)+\operatorname{con}(\Pi) .
$$

This formula was obtained on the assumption that $f$ is not identically zero on $\mathcal{O}$. In the general case we have to work through all the orbits and take their union. At this point we note that it is possible not even to consider the orbits, but just to take the sets $\{\phi \in \Phi(V) \mid h(\phi)>0\}$ for all dominant $h$. Moreover, the collection of these sets is finite, and we may take only the maximal sets of this type. Thus we have proved the following theorem. 
Theorem 7. We have

$$
\operatorname{Bri}\left(\overline{G\left\{v \mid \operatorname{supp}(v) \subset M_{h}\right\}}\right) \subset \operatorname{conv}\left(M_{h}\right)+\operatorname{con}(\Pi),
$$

where

$$
M_{h}=\{\phi \in \Phi(V) \mid h(\phi)>0\}
$$

and

$$
\operatorname{Bri}(\mathcal{N}(V)) \subset \bigcup_{h \in \mathcal{C}} \operatorname{conv}\left(M_{h}\right)+\operatorname{con}(\Pi) .
$$

Furthermore, in the last formula only the maximal sets $M_{h}$ need to be considered.

Note that these bounds were obtained without any restriction whatsoever on the representation.

Now let the highest weight $\delta$ of the adjoint representation be contained in the set of weights of $V$. We immediately notice that this condition is equivalent to the condition "the highest weight of $V$ lies in the root lattice" except for one case, namely the situation when the highest weight of $V$ is the highest short root. In this case we can describe the inner boundary of the Brion polytopes of the irreducible components of the nullcone. We need the following lemma.

Lemma 3. Let the highest weight $\delta$ of the adjoint representation be contained in the set of weights of $V$, let the element $h \in \mathbb{E}$ be dominant, and let $\Gamma$ be a face of the polytope conv $M_{h}$, contained in (conv $\left.M_{h}\right)_{\text {int }}$ and intersecting the interior of the Weyl chamber. Then the directional subspace of the affine subspace spanned by $\Gamma$ intersects the cone con (П) only at the origin.

Proof. Suppose the contrary. Then there is a nonzero vector $v$ in this intersection. Choose a point $p \in \Gamma$ contained in the interior of the Weyl chamber. Then $p=\sum a_{i} \alpha_{i}$ and $v=\sum b_{i} \alpha_{i}$, where $a_{i}>0$ and $b_{i} \geq 0$. Note now that $\Pi \subset M_{h}$, and therefore $\sum a_{i} \leq 1$. Hence there is a nonnegative number $t$ with $\sum\left(a_{i}+t b_{i}\right)=1$. Let $p^{\prime}=p+t v$. Then $p^{\prime} \in \operatorname{conv} \Pi \subset \operatorname{conv}\left(M_{h}\right)$. Recall that $p$, and hence also $p^{\prime}$, lies in the face $\Gamma$ of $\operatorname{conv}\left(M_{h}\right)$. Hence $\alpha_{i}$ also lies this face, but then $p=p^{\prime}$. Thus we have $\Gamma \cap \mathcal{C} \subset \operatorname{conv}(\Pi)$, but then $v$ lies in the space spanned by the differences of the simple roots, which intersects con (П) only at the origin. Contradiction.

This lemma has three important consequences, the first of which is equivalent to it. Let $V, h, M_{h}$ and $\Gamma$ be as defined above. Then

Proposition 5.1. The generating hyperplane of the face $\Gamma$ has a strictly dominant normal vector, which we denote by $n(\Gamma)$.

Proposition 5.2. Among the weights of $\Gamma$ there are no neighbors.

Proof. An immediate consequence of the fact that $\Delta^{+} \subset \operatorname{con}(\Pi)$.

Let $C_{h}=\mathbb{R}_{\geq 1} \cdot\left(\operatorname{conv}\left(M_{h}\right) \cap \mathcal{C}\right)$.

Proposition 5.3. We have the equality

$$
\left(\operatorname{conv}\left(M_{h}\right)+\operatorname{con}(\Pi)\right) \cap \mathcal{C}=C_{h} .
$$

Proof. The inclusion in one direction is clear, since for any $x$ belonging to $C_{h}$ there is a point $y$ of $\mathcal{C} \cap M_{h}$ such that $x \in \mathbb{R}_{\geq 1} y$, but then $x \in y+\operatorname{con}(\Pi)$. We now prove the reverse inclusion. Let $x \in\left(M_{h}+\operatorname{con}(\Pi)\right) \cap \mathcal{C}$. In order to show that $x \in C_{h}$ it suffices to verify that the condition $(x, n(\Gamma)) \geq(\Gamma, n(\Gamma))$ holds for all $\Gamma$ as in Lemma 3 . Represent $x$ in the form $y+\sum_{i} c_{i} \alpha_{i}$ where $y \in M_{h}$ and $c_{i} \geq 0$. Then by Proposition 5.1 we have $(x, n(\Gamma)) \geq(y, n(\Gamma))$, which also implies that the required conditions are satisfied. 
An immediate consequence of these propositions is the following theorem.

Theorem 8. Let the highest weight $\delta$ of the adjoint representation be contained in the set of weights of $V$, and let $X$ be an irreducible subset of the nullcone of the form $\bigcup_{v}$, where $\operatorname{supp}(v) \subset M_{h} G v$. Then the inner boundary of $\operatorname{Bri}(X)$ coincides with conv $\left(M_{h}\right)$ $\cap \mathcal{C}$.

Proof. The bound in one direction has already been proved, so it remains only to construct this set in the image of the moment map. For this it suffices to apply Proposition 2.2 to all the sets $\Gamma \cap \Phi(V)$.

Let

$$
R=\left(\left(\bigcup_{h} \mathbb{R}_{\geq 1} \operatorname{conv}\left(M_{h}\right)\right) \cap \mathcal{C}\right)_{\text {int }} .
$$

The bound we have obtained is an inner bound relative to $R$.

As we have just shown, $R \subset \operatorname{Bri}(\mathcal{N}(V))$, which gives us a description of the inner boundary of the Brion polytope for the nullcone.

Theorem 9. Let the highest weight $\delta$ of the adjoint representation be contained in the set of weights of $V$. Then the inner boundary of $\operatorname{Bri}(\mathcal{N}(V))$ coincides with $R$.

In the case of the adjoint representation we can now give an explicit description.

Theorem 10. The inner boundary of the Brion polytope for the nullcone of the adjoint representation has the form $\operatorname{conv}(\Pi) \cap \mathcal{C}$.

Proof. By definition in this case there is one possible set $M_{h}$, namely conv $\left(\Delta^{+}\right)$. Using Proposition 5.3 we obtain that $\operatorname{Bri}(\mathcal{N}) \subset \mathbb{R}_{\geq 1} \operatorname{conv}(\Pi) \cap \mathcal{C}$. It remains to verify that $\operatorname{conv}(\Pi) \cap \mathcal{C} \subset \operatorname{Bri}(\mathcal{N}(V))$. However, this follows from the fact that simple roots are not adjacent weights.

In the case of the adjoint representation the Brion polytope is convex, and so combining this theorem with Theorem 3 we obtain a description of the Brion polytopes for the nullcones of the adjoint representations of all simple algebras except $E_{6}$ and $A_{n \geq 4}$.

In the case of a representation other than the adjoint representation the Brion polytope for the nullcone need not be convex (and most often is not), so knowledge of the inner and outer boundaries are insufficient to describe it completely and we need additional theorems. The following section is devoted to this issue.

\section{Description of $\operatorname{Bri}(\mathcal{N}(V))$}

We now turn to the problem of describing the Brion polytope for the nullcone. In the previous two sections we obtained descriptions of the outer and inner boundaries respectively of the Brion polytope. Since this polytope may be nonconvex, these data are insufficient for a complete description and we need stronger conditions.

Let all the indices of the highest weight be at least two. We use the notation $A_{I}$ introduced earlier for the vertices of the intersection of the Weyl chamber with the polytope of weights of the representation.

Let $\# I=1$, that is $A_{I}=c \pi_{i}$ for some $i \in\{1, \ldots, n\}$ and $c \in \mathbb{Q}$. After scalar multiplication of equation (4.1) by $\pi_{i}$, we obtain $c_{i, i} c_{i}=\left(\lambda, \pi_{i}\right)$ where $c_{(j, i)}$ is the inverse transpose of the Cartan matrix. Recall that $m_{i} \geq 2$, which implies $c_{i} \geq \frac{\left(2 \rho, \pi_{i}\right)}{c_{i, i}}$.

Now for each algebra we define a certain vector $\alpha$. For $A_{n}$ and $C_{n}$ let

$$
\alpha=\sum_{1 \leq i \leq n} \alpha_{i}
$$


for $B_{n}$ with $n>2$

$$
\alpha=\sum_{1 \leq i \leq n-1} \alpha_{i}+\frac{\alpha_{n}}{2}
$$

for $F_{4}$

$$
\alpha=\frac{\alpha_{1}}{3}+2 \frac{\alpha_{2}}{3}+\alpha_{3}+2 \frac{\alpha_{4}}{3}
$$

for $E_{6}$

$$
\alpha=\frac{\alpha_{1}}{3}+2 \frac{\alpha_{2}}{3}+\alpha_{3}+2 \frac{\alpha_{4}}{3}+\frac{\alpha_{5}}{3}+\frac{\alpha_{6}}{3} ;
$$

for $E_{7}$

$$
\alpha=\frac{\alpha_{1}}{4}+\frac{\alpha_{2}}{2}+3 \frac{\alpha_{3}}{4}+\alpha_{4}+3 \frac{\alpha_{5}}{4}+\frac{\alpha_{6}}{2}+\frac{\alpha_{7}}{2} ;
$$

for $E_{8}$

$$
\alpha=\frac{\alpha_{1}}{3}+\frac{\alpha_{2}}{2}+2 \frac{\alpha_{3}}{3}+5 \frac{\alpha_{4}}{6}+\alpha_{5}+2 \frac{\alpha_{6}}{3}+\frac{\alpha_{7}}{3}+\frac{\alpha_{8}}{2} ;
$$

and for $G_{2}$ let

$$
\alpha=\alpha_{1}+\frac{\alpha_{2}}{2} .
$$

Denote by $d_{i}$ the coefficients of the decomposition of $\alpha$ in terms of the $\alpha_{i}$.

We study the scalar products $\left(A_{I}, \alpha\right)$. The point $A_{I}$ is the solution of the equation (4.1), and

$$
\left(A_{I}, \alpha\right)=\sum_{i \in I} d_{i}\left(A_{I}, \alpha\right)=\sum_{i \in I} d_{i} c_{i} .
$$

We now proceed as follows: we multiply equation (4.1) by $\pi_{i}$ for all $i \in I$, and obtain a system of linear equations of the form

$$
\sum_{i \in I} c_{j, i} c_{i}=\left(\lambda, \pi_{i}\right) \quad \forall j \in I .
$$

Multiplying the numbers $c_{i}$ by $d_{i}$ and the columns of the matrix $c_{(j, i)}$ by $\frac{1}{d_{i}}$ we obtain a new equation

$$
\sum_{i \in I}\left(\frac{c_{j, i}}{d_{i}}\right)\left(d_{i} c_{i}\right)=\left(\lambda, \pi_{i}\right) \text { for all } j \in I .
$$

After this transformation the matrix on the left-hand side has the following property: in any row the maximal element will be the diagonal one. The author is unaware of a general proof of this fact, but it can be easily verified by taking all the matrices $c_{(j, i)}$ (they can be found in [1, for example) and multiplying the columns by $\frac{1}{d_{i}}$.

We divide the rows of the matrix and the right-hand side by the diagonal elements. Corresponding to the above, the matrix on the left-hand side will have ones on the diagonal, and positive (but $\leq 1$ ) numbers off the diagonal. On the right-hand side are the numbers $\frac{d_{i}\left(\lambda, \pi_{i}\right)}{c_{i, i}}$. Hence for every $j \in I$ we have

$$
\left(A_{I}, \alpha\right)=\sum_{i \in I} d_{i} \alpha_{i} \geq \sum_{i \in I} \frac{c_{j, i} / d_{j}}{c_{j, j} / d_{j}} d_{i} \alpha_{i}=\frac{d_{j}\left(\lambda, \pi_{j}\right)}{c_{j, j}} \geq \frac{d_{j}\left(2 \rho, \pi_{j}\right)}{c_{j, j}} .
$$

Thus we have proved:

Lemma 4. Let all the indices of the highest weight be at least two. Then for any vertex $A_{I}$ with $I \neq \emptyset$ we have the inequality

$$
\left(A_{I}, \alpha\right) \geq b_{i}:=\max _{i \in I} \frac{d_{i}\left(2 \rho, \pi_{i}\right)}{c_{i, i}} .
$$


TABLE 2 .

\begin{tabular}{|l|c|c|c|c|c|c|c|c|}
\hline$A_{n}$ & $n+1$ & $n+1$ & $\ldots$ & $\ldots$ & $\ldots$ & $\ldots$ & $n+1$ & $n+1$ \\
\hline$B_{n}$ with $n>2$ & $2 n-1$ & $2 n-2$ & $\ldots$ & $\ldots$ & $\ldots$ & $\ldots$ & $n+1$ & $n$ \\
\hline$C_{n}$ & $2 n$ & $2 n-1$ & $\ldots$ & $\ldots$ & $\ldots$ & $\ldots$ & $n+2$ & $n+1$ \\
\hline$D_{n}$ & $2 n-2$ & $2 n-3$ & $\ldots$ & $\ldots$ & $n+2$ & $n+1$ & $n-1$ & $n-1$ \\
\hline$E_{6}$ & 4 & 6 & $14 / 3$ & 6 & 4 & $22 / 3$ & & \\
\hline$E_{7}$ & $9 / 2$ & $13 / 2$ & $15 / 2$ & 8 & $33 / 4$ & $17 / 2$ & 7 & \\
\hline$E_{8}$ & $29 / 3$ & 12 & $28 / 3$ & $55 / 6$ & 9 & $26 / 3$ & $23 / 3$ & $17 / 2$ \\
\hline$F_{4}$ & $11 / 3$ & $14 / 3$ & 5 & $16 / 3$ & & & & \\
\hline$G_{2}$ & 5 & 6 & & & & & & \\
\hline
\end{tabular}

The numbers $b_{i}$ on the right-hand side can be written out explicitly, since we defined the $d_{i}$ ourselves, and the $\left(2 \rho, \pi_{i}\right)$ and $c_{i, i}$ can be found in [1]. The data obtained are given in Table 2

We are now ready to prove the main theorem of this section.

Theorem 11. Suppose all the indices $m_{i}$ of the highest weight are at least two, and the highest weight lies in the root lattice. Then the Brion polytope for the nullcone is

$$
P=\left(\bigcup_{h} \mathbb{R}_{\geq 1} \operatorname{conv}\left(M_{h}\right)\right) \cap M(V) \cap \mathcal{C}
$$

Proof. Applying Theorem 7 and then Proposition 5.3, we obtain that the Brion polytope lies in the set $P$ just described. Thus we have to construct vectors that give $P$ in the image of the moment map. We begin by constructing vectors such that the union of their supports covers $P$, and then we verify that they have no adjacent weights. In fact we shall not even construct the vectors but will simply take the sets of weights bounded away from zero. We split $P$ into two parts, $P_{1}=P \cap \mathbb{R}_{\leq 1} \operatorname{conv}(\Pi)$ and $P_{2}=P \cap \mathbb{R}_{\geq 1} \operatorname{conv}(\Pi)$. First we construct sets of weights whose convex hulls cover $P_{1}$. For these we may take sets of the form $(\Gamma \cap \Phi(V)) \cup\left\{x_{i} \pi_{i}\right\}$, where $\Gamma$ is as defined in Lemma 3, and $x_{i}$ are the maximal numbers for which $x_{i} \pi_{i}$ is a weight of $V$. As is easily seen, the convex hulls of these sets cover $P_{1}$; we have to verify that in each such set there are no adjacent weights. In fact Proposition 5.2 implies that among the weights of the face $\Gamma$ there are no adjacencies. Furthermore, the weights of $\Gamma$ do not lie in the interior of the cone $\operatorname{con}(\Pi)$, and it is enough to check that $x_{i} \pi_{i}+\Delta$ lies in con $(\Pi)$. This last means that $x_{i} c_{i, j} \geq d_{j}$ for all $j$, where the weight $\sum d_{j} \alpha_{j}$ is the highest root. We now note that the $x_{i}$ can be estimated using Lemma 4 , and they turn out to be large enough (case-by-case verification). For example, for $A_{n}$ the fact that $b_{i} \geq n+1$ implies that $x_{i} \geq n+1$, that is, $x_{i} c_{i, j} \geq(n+1)(1 / n+1)=1=d_{i}$.

We next turn to $P_{2}$. According to the estimates obtained, this set lies in the convex hull of the points $A_{I}$ for $I \neq \emptyset$ and conv $(\Pi) \cap \mathbb{Q}_{+} \pi_{i}$. We now proceed as follows. Consider the set of weights $\chi$ that satisfy $(\chi, \alpha)>0$ and add to it the weights $\alpha_{i}$. Note that all the weights $\alpha_{i}$ satisfy $(\chi, \alpha) \geq 0$. Moreover, the collection of weights $\left\{\alpha_{i} \mid\left(\alpha_{i}, \alpha\right)=0\right\}$ forms a system of simple roots among the roots orthogonal to $\alpha$. Hence we can "slightly 
move" the vector $\alpha$ and replace it by $\alpha^{\prime}$ which will satisfy the following condition:

$$
\left(\chi, \alpha^{\prime}\right)>0 \Leftrightarrow\left[\begin{array}{l}
(\chi, \alpha)>0, \\
\left\{\begin{array}{l}
(\chi, \alpha)=0, \\
\chi \in \operatorname{con}\left\{\alpha_{i} \in \Pi \mid\left(\alpha_{i}, \alpha\right)=0\right\} .
\end{array}\right.
\end{array}\right.
$$

We fix an arbitrary one of these vectors $\alpha^{\prime}$.

It is well known that $X:=G\left\{v \mid\left(\operatorname{supp} v, \alpha^{\prime}\right)>0\right\}$ is an irreducible set lying in the nullcone. Hence $\operatorname{Bri}(X)$ is a convex set. We have to show that the vertices of $\operatorname{Bri}(X)$ are precisely the points $A_{I}$ with $I \neq \emptyset$ and $\operatorname{conv}(\Pi) \cap \mathbb{Q}_{+} \pi_{i}$. To prove the latter points lie in $\operatorname{Bri}(X)$ is easy. Indeed, it is enough to apply Lemma 2.2 to $\Pi$. Our problem now is to construct for every vertex $A_{I}$ a set $\Phi_{I}$ of $V$ such that firstly $A_{I} \in \operatorname{conv}\left(\Phi_{I}\right)$, secondly it has no adjacent weights, and thirdly $\left(\Phi_{I}, \alpha\right)>0$.

To each such vertex $A_{I}$ there corresponds a regular subalgebra $\mathfrak{g}_{I}$, a subset of the simple roots $\left\{\alpha_{i} \in \Pi \mid i \notin I\right\}$, a face $\Gamma_{I}$ and a representation $V_{I}$. Let $\mathfrak{g}_{I}: V_{I}^{\prime}$ be the restriction of the representation $\mathfrak{g}_{I}+\mathfrak{t}: V_{I}$ to $\mathfrak{g}_{I}$. The highest weight of this representation is obtained from the highest weight of $V$ as follows: if $\lambda=\sum_{i \in \Pi} m_{i} \pi_{i}$, then $\lambda_{I}=$ $\sum_{i \notin I} m_{i}\left(\pi_{I}\right)_{i}$. Let $W_{I}$ be the corresponding Weyl group.

Our problem is now to construct for every regular subalgebra of $\mathfrak{g}$ a set $\Phi_{I}^{\prime}$ of nonadjacent weights containing 0 in its convex hull. Let us fix $A_{I}$ and denote by $\varepsilon_{i}^{\prime}, \alpha_{i}^{\prime}$, $\pi_{i}^{\prime}$ the weights of the algebra $\mathfrak{g}_{I}$. We shall construct $\Phi_{I}$ as $A_{I}+\Phi_{I}^{\prime}$, taking into account the fact that all the indices of the highest weight are at least two.

Let $\mathfrak{g}_{I}=A_{1}$. Then either $0 \in \Phi\left(V_{I}\right)$, and we may take $\Phi_{I}^{\prime}=\{0\}$, or not, in which case we let $\Phi_{I}^{\prime}=\left\{-\pi_{1}, 3 \pi_{1}\right\}$.

Let $\mathfrak{g}_{I}=A_{n}$ with $n>1$. Then it is easy to verify that the collection of weights $0, \pi_{i}^{\prime}+\pi_{j}^{\prime}$ represents all factor classes of the weight lattice modulo the root lattice, and hence at least one of the weights is a weight of $V_{I}^{\prime}$. If $i=j$ we let $\Phi_{I}^{\prime}=W_{I}\left(2 \pi_{i}^{\prime}\right)$, and otherwise $\Phi_{I}^{\prime}=Z_{I}\left(\pi_{i}^{\prime}+\pi_{j}^{\prime}\right)$ where $Z_{I}$ is the subgroup of the Weyl group consisting of cyclic permutations.

Let $\mathfrak{g}_{I}=B_{2}$. We take the sets $\{0\}$ or $\left\{\pi_{1}^{\prime}+\pi_{2}^{\prime},-\pi_{1}^{\prime}-\pi_{2}^{\prime}\right\}$.

Let $\mathfrak{g}_{I}=B_{n}$ with $n>2$. Take $\{0\}$ or $\left\{\pi_{n}^{\prime},-\pi_{n}^{\prime}\right\}$.

Let $\mathfrak{g}_{I}=C_{n}$ with $n>2$. Take $\{0\}$ or $\left\{\pi_{3}^{\prime},-\pi_{3}^{\prime}\right\}$.

Let $\mathfrak{g}_{I}=D_{n}$ with even $n>3$. Take

$$
\{0\},\left\{\pi_{1}^{\prime}+\pi_{2}^{\prime},-\pi_{1}^{\prime}-\pi_{2}^{\prime}\right\},\left\{\pi_{n}^{\prime},-\pi_{n}^{\prime}\right\} \text { and }\left\{\pi_{n-1}^{\prime},-\pi_{n-1}^{\prime}\right\} .
$$

Let $\mathfrak{g}_{I}=D_{n}$ with odd $n>3$. Take

$$
\{0\},\left\{\pi_{1}^{\prime}+\pi_{2}^{\prime},-\pi_{1}^{\prime}-\pi_{2}^{\prime}\right\}, Z_{I}\left(\pi_{1}^{\prime}+\pi_{n}^{\prime}-\varepsilon_{n-1}^{\prime}-\varepsilon_{n}^{\prime}\right) \text { and } Z_{I}\left(\pi_{2}^{\prime}+\pi_{n}^{\prime}-\varepsilon_{n-1}^{\prime}-\varepsilon_{n}^{\prime}\right) \text {. }
$$

Let $\mathfrak{g}_{I}=E_{6}$. Take $\{0\}, \mathcal{W}_{I}\left(2 \pi_{1}^{\prime}\right)$ or $\mathcal{W}_{I}\left(2 \pi_{2}^{\prime}\right)$.

Let $\mathfrak{g}_{I}=E_{7}$. Take $\{0\}$ or $Z_{I}\left(\pi_{1}\right)$.

Let $\mathfrak{g}_{I}=F_{4}, G_{2}$ or $E_{8}$. In all cases take $\{0\}$.

Thus in all cases when the algebra $\mathfrak{g}_{I}$ is irreducible we find that

$$
\forall \phi \in \Phi_{A}^{\prime} \quad \exists \psi=-\pi_{i}^{\prime}-\pi_{j}^{\prime}: \phi \in \psi+\operatorname{con}\left(\Pi_{I}\right),
$$

and for the case $A_{1}$ a stricter condition is satisfied: we can discard $\phi_{j}^{\prime}$.

If $\mathfrak{g}_{I}$ is reducible then any weight of $V_{I}$ can be expressed as a sum of weights of the spaces $V_{J}$, where $J$ runs through the sets corresponding to the irreducible components 
of $\mathfrak{g}_{I}$. We define $\Phi_{I}^{\prime}$ to be the set of sums of weights lying in the $\Phi_{J}^{\prime}$. Next note that $-\pi_{i}^{\prime} \in-\pi_{i}+\operatorname{con}_{j \in I}\left(\pi_{j}\right)$, and so we obtain the following result: suppose $\mathfrak{g}_{I}$ has $a$ simple components of type $A_{1}$ and $b$ others. Then

$$
\left(\Phi_{A}, \alpha\right)>\max _{i \in I} b_{i}-\max _{i \notin I} d_{i}(a+2 b) .
$$

Thus it remains to prove that the right-hand side of this formula is nonnegative in all cases.

Let $\mathfrak{g}=A_{n}$. Then we obtain the condition $n+1>(a+2 b)$, and this is satisfied because $a+2 b \leq \# \Pi=n$. For $B_{n}$ and $C_{n}$ the proof is similar, since $b_{i} \geq n$. Let $\mathfrak{g}=D_{n}$. Note that in this case $a+b \leq \# I+2$. Hence $2 a+3 b \leq n$, and $a+2 b \leq n+1$. Now let $\mathfrak{g}=F_{4}$. Since $\# I \geq 1$, we obtain that $a+2 b \leq 3$, but all $d_{i} \leq 1$, and $b_{i}>3$. Let $\mathfrak{g}=E_{6}$. It is easy to see that $a+b \leq 3$, where even in this case $a \geq 2$, that is $a+2 b \leq 4$. And as we know, $b_{i} \geq 4$. Let $\mathfrak{g}=E_{7}$. It is easy to verify that $a+b \leq 4$, and if $a+b=4$, then $4 \in I$, that is $\max _{i \in I} b_{i}=8$. Let $\max _{i \in I} b_{i}<6$; then $a+2 b=2$. In the remaining cases $\max _{i \in I} b_{i} \geq 6$ and $a+2 b \leq 6$. Let $\mathfrak{g}=E_{8}$. Then it is easy to check that $b_{i} \geq 7$, and $a+2 b \leq 6$. Let $\mathfrak{g}=G_{2}$. Then $a+2 b \leq 1$, and the numbers $b_{i}$ are larger.

This case-by-case analysis proves the required condition.

\section{REFERENCES}

[1] E. B. Vinberg and A. L. Onishchik, A seminar on Lie groups and algebraic groups, Second edition, URSS, Moscow, 1995. (Russian) MR1403378 (97d:22001)

[2] E. B. Vinberg and V. L. Popov, Invariant theory. Itogi Nauki Tekh., Ser. Sovrem. Probl. Mat., Fundam. Napravleniya 55 (1989), 137-314. English transl., Encyclopaedia Math. Sci. vol 55, Springer, 1994. MR.1100485 (92d:14010)

[3] A. G. Elashvili, Canonical form and stationary subalgebras of points of general position for simple linear Lie groups, Funkts. Anal. Prilozh. 6 (1972), 51-62. English transl., Funct. Anal. Appl. 6 (1972), 44-53. MR0304554 (46:3689)

[4] A. G. Elashvili, Stationary subalgebras of points of the common state for irreducible linear Lie groups, Funkts. Anal. Prilozh. 6 (1972), 65-78. English transl., Funct. Anal. Appl. 6 (1972), 139148. MR0304555|(46:3690)

[5] M. Atiyah, Convexity and commuting Hamiltonians, Bull. London Math. Soc. 14 (1982), 1-15. MR.0642416 (83e:53037)

[6] M. Brion, On the general faces of the moment polytope, Int. Math. Res. Not. 1999, 185-201. MR.1677271 (2000i:14068)

[7] M. Brion, Sur l'image de l'application moment, Sém. d'Algébre P. Dubreil et. M.-P. Malliavin Proc., Paris. 1986. Lecture Notes in Math., vol. 1296, pp.177-192, Springer-Verlag, 1987. MR0932055 (89i:32062)

[8] M. Brion, D. Luna, and Th. Vust, Espaces homogènes sphériques, Invent. Math. 84 (1986), 617-632. MR0837530 (87g:14057)

[9] B. Broer, Hilbert series in invariant theory, Thesis. Rejksuniversiteit Utrech, 1990.

[10] W. Fulton, Young Tableaux, London Mathematical Society Student Texts, 35. Cambridge University Press, 1997. MR 1464693 (99f:05119)

[11] F. Grosshans, Constructing invariant polynomials via Tschirnhaus transformations, in: Invariant theory (S. S. Koh, ed.) Lecture Notes in Math., vol. 1278, pp. 95-102, Springer-Verlag, 1987. MR.0924168 (89a:14066)

[12] J. Humphreys, Introduction to Lie algebras and representation theory, Graduate Texts in Mathematics, Vol. 9. Springer-Verlag, New York, 1972. MR0323842 (48:2197)

[13] W. H. Hesselink, Characters of the nullcone, Math. Ann. 252 (1980), 179-182. MR0593631 (82c:17004)

[14] B. Kostant, Lie group representations on polynomial rings, Amer. J. Math. 85 (1963), 327-404. MR.0158024(28:1252)

[15] A. J. Macfarlane and H. Pfeiffer, Representations of the exceptional and other Lie algebras with integral eigenvalues of the Casimir operator, J. Phys. A 36 (2003), 2305-2317. MR1965160 (2004f:17016) 
[16] V.L. Popov, Moment polytopes of nilpotent orbit closures; dimension and isomorphism of simple modules; and variations on the theme of J. Chipalkatti, in Invariant Theory in All Characteristics (Proc. Workshop on Classical Invariant Theory, Queen's University, Ontario), H. E. A. Campbell and D. L. Wehlau (eds.), CRM Proc. and Lecture Notes 35, AMS 2004. MR2066466

[17] R. Sjamaar, Convexity properties of the moment mapping reexamined. Adv. in Math. 138 (1998), 46-91. MF,1645052(2000a:53148)

Moscow State University, Faculty of Mechanics and Mathematics, Moscow 119899, Russia

E-mail address: asmirnov@rdm.ru

Translated by D. R. J. CHILLINGWORTH 